\title{
Forces between oil drops in polymer-surfactant systems: linking direct force measurements to microfluidic observations
}

Emily J. Jamieson, Christopher J. Fewkes, Joseph D. Berry, Raymond R. Dagastine

Department of Chemical Engineering and the Particulate Fluids Processing Centre, University of Melbourne, Parkville, VIC 3010, Australia

Author E-mail Addresses:

Emily Jamieson: e.jamieson@student.unimelb.edu.au

Christopher Fewkes: christopher.j.fewkes@gmail.com

Joseph Berry: joe.d.berry@gmail.com

Raymond Dagastine: rrd@unimelb.edu.au

Corresponding Author:

Raymond R. Dagastine

Department of Chemical Engineering and the Particulate Fluids Processing Centre, University of Melbourne, Parkville, VIC 3010, Australia

rrd@unimelb.edu.au

T: +61-3-8344-4704

F: $+61-3-8344-4153$

\section{Abstract}

Both microfluidics and atomic force microscopy have been used to quantify the forces between oil drops in the presence of complexes formed with anionic surfactant, sodium dodecyl sulphate, and neutral, water soluble polymer, poly(vinylpyrrolidone). Measurement and modelling of the interaction forces between both rigid and deformable surfaces demonstrated that the attraction between the drops is due to depletion forces, whereas the repulsive force is a combination of electrical double layer and steric forces, indicating complexes exist both in the bulk and at the drop interface. We developed a new microfluidic device to first form and then break-up chains of drops, where the drop break-up is sensitive to the underlying surface forces between the drops, not hydrodynamic drainage forces. The interaction behaviour between the force measurements and the microfluidic observations showed a strong correlation, where the observed adhesion between drops in the microfluidics is sensitive to the drop deformation and Laplace pressure. Correlation between the two techniques provides insight into the surface forces between drops in flowing systems. This work opens the possibility of developing high-throughput measurements of adhesive interactions between drops and has the potential utility in the formulation of emulsions. 
Keywords

Microfluidics; Atomic force microscopy; Drops; Deformable; Hydrodynamics; Depletion forces; Surface forces

\section{Abbreviations}

AFM, atomic force microscopy; CMC, critical micelle concentration; PS, polymer-surfactant; SDS, sodium dodecyl sulphate; PVP, poly(vinylpyrrolidone); CAC, critical aggregation concentration; EDL, electrical double layer;

\section{Introduction}

Studies of interactions between drops and bubbles usually focus on the transition between repulsion and attraction, defining precise conditions whereby drops/bubbles may coalesce. These measurements are usually made using direct force measurement methods such as atomic force microscopy (AFM) (1-8), larger capillary based drop and bubble methods sensitive to interfacial separation (9-13), insightful and novel microfluidic devices (14-18), or other geometries yielding elongational flow such as the four roll mill (19). However, there are conditions under which drop/bubble interactions are attractive over certain separation distances yet sufficiently stable at closer separations such that aggregation occurs, rather than coalescence (20). For drop interactions in emulsions, these attractive interactions between the drops underpin the properties of these systems and control processes such as gel formation, fluid microstructure, and the deposition of coatings. This has important applications in the formulation and processing of foods, personal care products, and pharmaceuticals.

The term "adhesive emulsions", first coined by Bibbette (20), suggests that specific adhesive or attractive interactions are key to controlling or tuning the behaviour of these systems (21). At liquidliquid or liquid-gas interfaces these types of attractive interactions, often attributed to a range of surface forces including depletion and structural forces, were initially measured in systems of flat airwater and oil-water interfaces using thin film balances in the seminal work of Langevin (22-25), as well as Wasan's work using the Scheludko cell technique $(26,27)$. More recently, a series of AFM studies investigated aggregation in drop and bubble systems, as the AFM can probe both attractive and repulsive interactions, whereas thin film studies require a stable film for any observation. In addition, the AFM studies have elucidated the importance of the interplay between drop/bubble deformation and these structural or depletion forces in foams or emulsions $(5,28-32)$.

One class of adhesive emulsion systems, common to formulated products, is formed by the complexation of long-chained polymers with various surfactants $(25,33)$. Over a range of surfactant concentrations, often spanning from below to well above the critical micelle concentration (CMC), the surfactant and polymer form molecular complexes due to the association of the surfactant with the polymer. This association is based on interactions of the surfactant head group with the polar or hydrophilic regions of the polymer, as well as the hydrophobic association of the surfactant tail with the less polar or hydrophobic regions of the polymer (34-36). Thus, even for a hydrophilic polymer showing little surface activity, the polymer-surfactant (PS) complex is often surface-active over a significant range of surfactant concentrations (34, 37-39). The PS complex and resultant attractive interaction is influenced by many factors including surfactant concentration, added electrolytes, polymer backbone flexibility, polymer charge, and surfactant-polymer associations (often based on charge or hydrophobic interaction). This sensitivity and flexibility is one of the reasons why PS complexes are used so widely in formulated products (40). This has led to an extensive understanding of PS complex behaviour in the bulk $(25,37,39,41)$. However, there are still uncertainties related to 
the interaction between PS complexes and surfaces, summarised in numerous reviews on the topic $(22,25,42,43)$, and in particular the interactions between surfaces coated with the complexes. Thus, quantifying the forces involved between two interacting surfaces coated with PS complexes is important to understanding the fundamental nature of PS systems.

PS complexes coated on hydrophobic solid surfaces have been found to exhibit attractive adhesive forces between the surfaces due to polymer bridging or depletion forces (43-49). The characterisation of such systems is limited not only in the choice of surface, polymer and surfactant but concentration variation and the effect of added components. As mentioned above, thin film balance methods have been used to explore the forces between flat air-water interfaces where both bridging and stratification of the PS complex layers have been observed $(22-25,50,51)$, however these forces have not been explored with drop/bubble systems exhibiting curvature and deformation in the same detail. Depletion and structural forces have been previously studied in drop/bubble pair interactions using AFM in a number of systems where strong attractions are observed, but not coalescence. Gromer et al. measured the effect of a non-adsorbing polyelectrolyte on the structural forces between two oil drops in food systems, notably sugar beet pectin (52). Other studies using AFM have probed the behaviour of oil drop-pair interactions in the presence of surfactant micelles, microemulsions, nanoparticles and rod-like surfactants (30-32). In addition, the interaction of a bubble and a particle was studied in the presence of non-adsorbing nanoparticles (53), and structural and depletion forces in the presence of non-adsorbing polyelectrolytes was observed between bubble pairs for both monodisperse and polydisperse polyelectrolytes $(28,29)$. The detailed modelling in many of these studies showed that the interplay of the structural and depletion forces with the deformation of the drops/bubbles lead to significant attractive interactions at small separations not present with equivalent sized rigid particles. In these studies, the force behaviour showed a clear dependence on the Laplace pressure of the drops (32). However, PS complexes have not yet been explored using AFM with drops/bubbles. The only direct force measurements with nanometer resolution in separation we are aware of involving PS complexes are the elegant studies by Mondain-Monval and co-workers using the magnetic field induced chaining technique (MCT), which offers some insight into these systems, but are limited to femo-Newton forces, micron to sub-micron drops that exhibit little if any deformation and struggle to probe attractive forces (54).

PS complexes are often difficult to study in a direct force measurement context over a large surfactant concentration range as many PS complexes induce phase separation, especially when the polymer and surfactant are oppositely charged (46). The bulk phase behaviour of the common anionic surfactant sodium dodecyl sulphate (SDS) and non-ionic polymer poly(vinylpyrrolidone) (PVP) has been covered in detail throughout the literature $(35,37,39,55)$, and is used in this study as the complex is water soluble and does not induce phase separation $(56,57)$. It is understood that once SDS begins to bind to PVP it forms 'clusters' with aggregation numbers below that of a standard surfactant micelle (37, $55,58-60$ ). (See supplementary material for further details)

Interactions between SDS and PVP at an air-water interface have also been observed using several techniques such as pendant drop tensiometry and neutron scattering $(34,61,62)$. For neutral polymers and charged surfactants, it is widely accepted in the literature that the interfacial tension behaviour for a polymer-surfactant system with constant polymer concentration and varying surfactant concentration can be described by 3 regions, delineated by the points of discontinuity in the interfacial tension versus concentration curve. The initial change, denoted as $T_{1}$, is the result of the onset of surfactant aggregation to the polymer also known as the critical aggregation concentration (CAC) (63). At $\mathrm{T}_{2}$ the polymer is considered to be completely saturated, such that further addition of surfactant does not increase the amount on the interface or polymer, but instead results 
in the formation of surfactant only micelles $(36,55,58,64)$. This point occurs at interfacial tension values similar to those of a pure surfactant system at its $\mathrm{CMC}$, which is due to the availability of free surfactant molecules competing with the polymer-surfactant complex for adsorption at the interface (42). Observations from literature report CAC values for 0.5\% PVP and SDS in a SDS concertation range of $1-3 \mathrm{mM}$, by using a range of techniques, such as conductivity (65), surface tension measurements $(34,36)$ and capillary electrophoresis $(66)$. The saturation of the polymer is reported over a much larger SDS concentration range, from 15-25 mM, attributed to the dependence on polymer molecular weight and concentration (42).

PS complexes formed between neutral polymers and charged surfactants have been observed to behave as pseudo-polyelectrolytes (67-71). Light scattering experiments performed by Minatti et al. claimed PVP and SDS aggregates have properties similar to polyelectrolytes, however due to the multiple equilibria that exists between the molecules the system exhibits higher complexity to that of a standard linear polyelectrolyte (72). Furthermore, Wu et al. used capillary electrophoresis to present direct evidence that PVP and SDS complexes should be treated as pseudo-polyanions as the apparent electrophoretic mobility of the complex decreases with increasing SDS aggregation (73). This behaviour has also been accounted for in studies observing the force between surfaces in systems containing neutral polymers and anionic surfactants. Using AFM, Tulpar et al. measured strong depletion forces between a silica particle and plate in the presence of Pluronic F108/SDS complexes, which they regarded as large polyelectrolytes (48). The previously mentioned work by MondainMonval credited an increase in repulsive electro-steric forces between emulsion droplets in solutions containing PVA/SDS complexes to their polyelectrolyte nature (54). In addition, a single foam stability study by Folmer et al., using a thin film balance method, has observed stable films and stratification in the films with PVP/SDS at concentrations where they exhibit strong binding (50). To our knowledge there is a gap in the literature covering the direct forces between rigid or deformable surfaces in a $\mathrm{PVP} / \mathrm{SDS}$ system to probe the attractive forces in this system.

It is possible to investigate PS complexes or any emulsion system by examining the drop pair interactions using direct force measurement methods such as AFM, larger scale methods such as the Integrated Thin Film Drainage Apparatus (IFTDA)(9, 74$)$ or the cantilevered capillary apparatus (10, 75). However, these studies require extensive sub-discipline expertise and are unable to perform measurements at a timescale comparable to other emulsion characterisation methods. Thus, potential advancements can be made by developing methods that probe soft particle attraction and adhesive forces in a high-throughput context over a wide range of operating conditions. Microfluidic devices are an obvious candidate to systematically probe drop interactions, yet previous studies that have had such success have been limited to studying phenomena that are either dominated by hydrodynamic interactions between drops or exhibit some coupling of hydrodynamic behaviour and surface forces. The exciting studies by Bremond \& Bibbette, $(14,15)$ and Gunes et al.,(16, 17) systematically explored drop coalescence in microfluidic devices, most notably where drops coalesced upon separation. However, the drop interaction outcomes in these studies were driven largely by hydrodynamic drainage effects until very close drop separations. Furthermore, the seminal work by Anna (18) into drop coalescence at $T$ and $Y$ shaped junctions specifically noted that the hydrodynamics of the surrounding fluid dominated any interaction, effectively masking equilibrium surface forces. $A$ recent simulation study of drop and bubble collisions in AFM experiments, by Berry and Dagastine (1), mapped the rich phenomenological interplay between equilibrium surface forces, drop deformation and the hydrodynamic drainage effects, identifying collision velocities where hydrodynamic drainage effects play a small role in the collision outcomes. Thus, in order to study equilibrium surface forces with a microfluidic platform, it is necessary to create a geometry that removes or quantifiably controls the hydrodynamic interactions during soft particle collisions. An example of the latter can be seen in 
some recent work into microfluidic flow that used depletion forces to form drop aggregates, prior to photo-polymerisation, by controlling the channel flowrate through a channel expansion (76).

The current investigation focuses on the strong attractions between oil drops in the presence of complexes formed with anionic surfactant and neutral, water soluble polymers. Both direct force measurements between two oil drops measured with AFM and microfluidic observations of the formation and then breakup of chains of oil drops were studied via systematically varying surfactant concentration at constant polymer concentrations. The two techniques were used to better correlate the behaviour of droplets in flowing systems such as microfluidics with the underlying surface forces. Doing so works towards the possibility of developing high-throughput measurements of adhesive interactions between oil drops and has the potential to enable the rapid screening and optimal selection of new components and additives for advanced materials.

\section{Experimental Section}

\section{Materials}

Sodium dodecyl sulphate (SDS, >99\% (GC)) was obtained from Sigma-Aldrich (New South Wales, Australia) and used as received. Polyvinylpyrrolidone (PVP, K90, MW $=360,000 \mathrm{~g} / \mathrm{mol}$, analytical grade) was obtained from Fluka and used as received. Deionised water at $25^{\circ} \mathrm{C}$ (minimum resistivity 18.2 $\mathrm{M} \Omega \mathrm{cm}$ ) was taken from a Milli-Q system. Tetradecane (98\%) was obtained from Sigma-Aldrich (New South Wales, Australia) and purified over silica (Florisil, Sigma-Aldrich, 100-200 mesh) via column chromatography. No adjustments were made to solution $\mathrm{pH}$ or ionic strength.

\section{Interfacial Tension}

Interfacial tensions were measured using pendant drop tensiometry with a Dataphysics OCA 20 Tensiometer. This involved generating a drop of oil in the aqueous solution within a clear quartz cuvette, using an inverted needle as the oil $\left(721 \mathrm{~kg} / \mathrm{m} 3 @ 25^{\circ} \mathrm{C}\right)$ is less dense than water $(998.9$ $\mathrm{kg} / \mathrm{m} 3 @ 25^{\circ} \mathrm{C}$ ). The cell was sealed with Parafilm to avoid dust contamination with care taken to ensure the solutions did not come in contact with the Parafilm. The drops were generated manually by depressing a syringe attached to the needle. Once a drop of desired size was produced, the drop profile was photographed with the needle diameter used as a reference and then fit to the YoungLaplace equation using the proprietary instrument software based on the deformation of the drop and fluid properties input into the calculations. The balance between interfacial tension forces and buoyancy allowed for the interfacial tension to be calculated (77). To account for surface adsorption kinetics the interfacial tension was tracked over time until it was considered to be stable on a log-log scale.

\section{AFM}

All experiments were performed on an Asylum MFP-3D AFM. The procedure for drop-drop (deformable) and particle-slide (rigid) AFM measurements has been covered in detail in previous literature $(5,7,31,32)$. All slides were cleaned prior to use by soaking for $1 \mathrm{hr}$ in $10 \%$ Ajax detergent (LabChem), followed by $1 \mathrm{hr}$ in 10\% sodium hydroxide solution, then $1 \mathrm{hr}$ in $10 \%$ nitric acid (70\%, Univar) solution, and finally thoroughly rinsed with Milli-Q water. Spring constants were measured using the method of Hutter and Bechhoefer.(78)

Rigid AFM experiments used a triangular silicon nitride cantilever with a $5 \mu \mathrm{m}$ diameter $\mathrm{SiO}_{2}$ sphere attached at the tip (PT.SiO2.SN.5, Novascan, IA, USA), and a flat rigid glass round slide. The cantilevers used to measure the hydrophilic system were exposed to low concentration ozone (BioForce Nanosciences, Inc., UV/Ozone ProCleaner Plus) prior to use. To functionalise the surfaces for hydrophobic 
measurements, the glass slide and both sides of the cantilever were sputter coated (Emitech K575X) with a thin layer of chromium ( $7 \mathrm{~nm})$ followed by a layer of gold $(\sim 30 \mathrm{~nm})$ and then submerged in a $2 \mathrm{mM}$ decanethiol solution for a minimum of $2 \mathrm{hrs}$. The spring constants of these cantilevers were measured to be in the range of 0.03-0.05 $\mathrm{N} \mathrm{m}^{-1}$.

Drop experiments were performed using custom rectangular silicon cantilevers (450 $\mu \mathrm{m} \times 50 \mu \mathrm{m} \times 2$ $\mu \mathrm{m}$ ) with a gold disk (radius of $45 \mu \mathrm{m}$ ) at the end, which were fabricated at the Victorian node of the Australian Nanofabrication Facility (ANFF), the Melbourne Centre for Nanofabrication. $(32,79)$ These cantilevers were submerged in a dilute $2 \mathrm{mM}$ solution of decanethiol in ethanol to form a decanethiol monolayer on the gold disk to facilitate the drop attachment. A layer of chromium $(\sim 7 \mathrm{~nm})$ followed by gold ( $\sim 30 \mathrm{~nm}$ ) was than sputter coated (Emitech K575X) onto the backside of the cantilever to ensure a sufficient laser signal was registered by the photodiode during AFM measurements. The spring constants of these cantilevers were in the range of 0.1-0.3 $\mathrm{N} \mathrm{m}^{-1}$. As the oil is less dense then water it was necessary to make the glass round hydrophobic in order to immobilise the drops on the surface of the slide. This was achieved by boiling the slides in $\mathrm{n}$-propanol for 2-5 $\mathrm{h}$ prior to use where the cantilevers were more hydrophobic then the substrate to aide drop attachment. Drops were generated by spray injection using a deformed hypodermic needle and submerged in solution using an O-ring technique outlined by Dagastine et al.(80). Drop radii were measured before and after a set of experiments using optical microscopy. Raw data was recorded as photodiode voltage versus linear variable differential transformer (LVDT) position. Force curves were taken at a scan speed of 100 and $200 \mathrm{~nm} \mathrm{~s}^{-1}$ unless otherwise stated.

Force Curve Analysis:

Rigid Surfaces

Force versus piezo motion data between two rigid silica surfaces in the SDS-PVP system were converted to force versus separation data by using the constant compliance region, the measured photodiode sensitivity and cantilever spring constant. These data were fitted to an analytical model for electrical double layer and depletion forces, where the total potential energy between two surfaces was modelled as the sum of these forces. For this study, the net interaction energy $E$ between flat half spaces as a function of surface separation $h$ is given by:

$$
E(h)=E_{E D L}+E_{D E P} .
$$

where $E_{E D L}$ is the electrical double layer (EDL) potential energy and $E_{D E P}$ is the depletion potential energy. Assuming a weak overlap model, the EDL potential energy between two planar surfaces with matching chemistry can be modelled as (81):

$$
E_{E D L}=\frac{64 k_{b} T n^{o}}{\kappa} \tanh \left(\frac{e \psi_{0}}{4 k_{b} T}\right)^{2} e^{-\kappa h},
$$

where $k_{b}$ is the Boltzmann constant, $\mathrm{T}$ is the absolute temperature, $n^{o}$ is the bulk ion number density, $\kappa$ is the inverse Debye length, $e$ is the elemental charge, and $\psi$ is the surface potential for each interacting surface. The depletion potential energy can be modelled with Asakura-Oosawa theory, according to (82): 


$$
E_{D E P}= \begin{cases}\Pi_{\mathrm{OSM}}(2 \Delta-h) & \text { for } h<2 \Delta \\ 0 & \text { for } h \geq 2 \Delta\end{cases}
$$

where $\Pi_{\text {OSM }}$ is the osmotic pressure and $\Delta$ is the depletion layer thickness. The Debye length and bulk ion number density for a given system can be calculated from the ionic strength. The Derjaguin approximation was used to relate the interaction energy of two flat surfaces to the force measured in the AFM measurement for a sphere and a flat plate. The fitted model was then used to determine the remaining unknown surface force parameters, the surface potential, osmotic pressure and depletion layer thickness, from the experimental data.

\section{Deformable Drops}

To quantitatively model the forces between two deformable surfaces we used a well-established theory that describes the interplay of changes in drop deformation, separation, surface forces and hydrodynamic drainage effects between drops/bubbles (83-88), sometimes referred to as the StokesReynolds-Young-Laplace (SRYL) model for drop and bubble interactions. This theory has been previously validated against measurements by both the authors and others for both equilibrium surface forces measurements and conditions where hydrodynamic drainage is significant $(6,89)$. The Reynolds lubrication approximation accounts for the thin film drainage between two drops or bubbles bought into close separation, describing the change in film thickness as a function of axial coordinate $r$ with:

$$
\frac{\partial h}{\partial t}=\frac{1}{12 \mu r} \frac{\partial}{\partial r}\left(r h^{3} \frac{\partial \mathrm{P}_{\mathrm{H}}}{\partial r}\right)
$$

where $\mu$ is the viscosity of the liquid in the film and $\mathrm{P}_{\mathrm{H}}$ is the hydrodynamic pressure in the film. The normal stress balance between the drop shape and disjoining pressure arising from the surface forces within the film is governed by a modified form of the Young-Laplace equation, with an additional term accounting for the effects of the hydrodynamic pressure:

$$
\frac{\gamma}{2 r} \frac{\partial}{\partial r}\left(r \frac{\partial h}{\partial r}\right)=\frac{2 \gamma}{R}-\Pi(\mathrm{h})-P_{H}(h)
$$

Here $\gamma$ is the interfacial tension of the system, $R=\left(2 R_{1} R_{2}\right) /\left(R_{1}+R_{2}\right)$ is the effective radius of the two drops and $\Pi$ is the disjoining pressure. The force of interaction between the drops is defined by:

$$
F(t)=2 \pi \int_{o}^{\infty}\left[\Pi(\mathrm{h})+P_{H}(h)\right] r d r
$$

In flow regimes where the system is considered to be in equilibrium such that hydrodynamic drainage is negligible, the system is modelled by the Young-Laplace equation without the hydrodynamic pressure contribution. In both cases, the disjoining pressure is defined as the negative of the derivative of the $E(h)$ :

$$
\Pi(h)=-\frac{\partial E(h)}{\partial h}
$$


The governing equations can be solved using appropriate boundary conditions for both equilibrium and dynamic cases to calculate the expected force measured using the AFM. These boundary conditions and further details of the modelling can be found in the Supplementary Material.

\section{Microfluidics}

The microfluidic devices were manufactured from borosilicate glass by the South Australia node of ANFF using deep reactive ion etching (DRIE). The experiments were conducted on an inverted optical microscope (XDS-2 Inverted Biological Microscope, Daintree Scientific, St. Helens, Tasmania, Australia). The syringes used for these experiments were $500 \mu \mathrm{L}$ glass (Hamilton Company), and 100 $\mu \mathrm{L}$ glass (Hamilton Company) luer lock syringes. The $500 \mu \mathrm{L}$ syringes were used for the fluid injection channels and the $100 \mu \mathrm{L}$ syringes were used for the withdraw channels, as this needed very fine control and does not have problems with fluid dead zones or water in the line. Syringes were driven by fluid pumps (NE-1000, NE-3000, New Era Pump Systems, New York, United States of America) that were controlled manually.

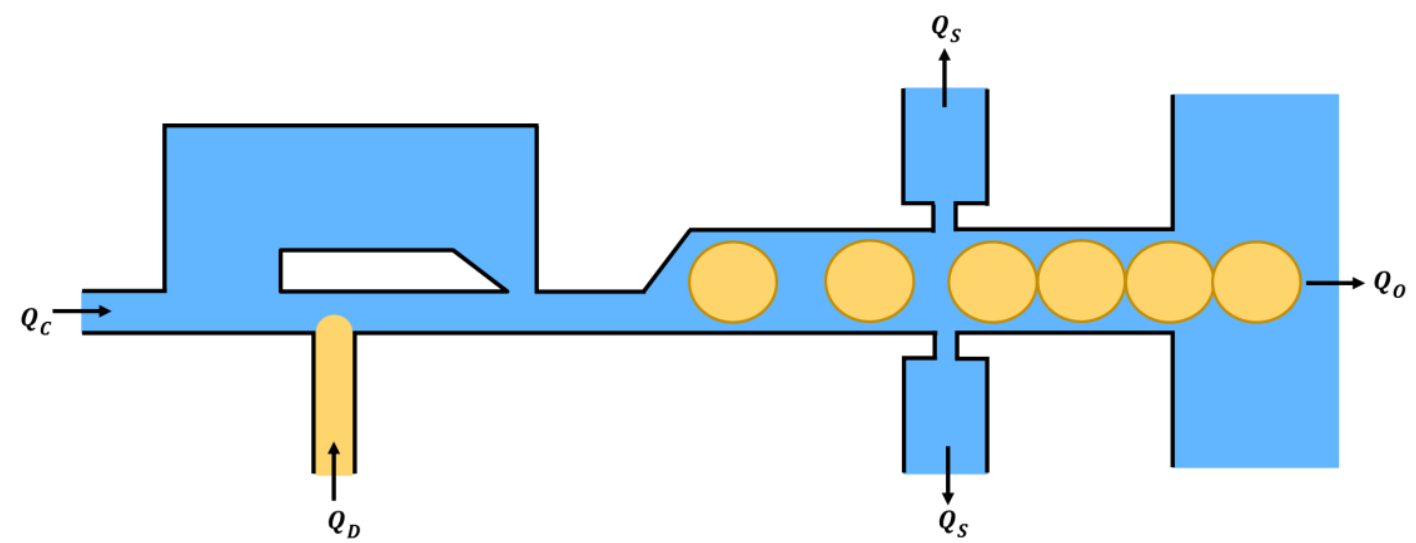

Figure 1 Simplified top down schematic diagram of the microfluidic geometry designed to generate drop chains. A block and break junction is used to generate the drops and then two side channels are used to collide and bunch the drops together by removing the excess continuous phase. The droplet chains then flow into solution. Flowrates were set on the fluid pumps as $Q_{C}=30 \mu \mathrm{L} / \mathrm{hr}, Q_{D}=10 \mu \mathrm{L} / \mathrm{hr}$, and $\mathrm{Q}_{\mathrm{S}}=11 \mu \mathrm{L} / \mathrm{hr}$, resulting in $\mathrm{Q}_{0}=18 \mu \mathrm{L} / \mathrm{hr}$, giving drop with an equivalent spherical radius of $\sim 28 \mu \mathrm{m}$ and a drop velocity of $\sim 2 \mathrm{~mm} / \mathrm{s}$.

Figure 1 demonstrates a simplified schematic for the drop chaining microfluidic device with drop generation, collision, and release. This device combines a block and break junction for droplet generation with two side channels to initiate drop collisions by removing excess continuous phase (90). A detailed schematic outlining channel dimensions can be found in the supplementary material (See S1). The apparatus was designed such that the drops exited the microfluidic device into solution within a large Petri-dish as shown in Figure S2. To avoid drop build-up at the exit, and to demonstrate the existence of an attractive force between drops as a resistance to drop separation in the fluid flow, an external flow was generated near the exit of the microfluidic device. This was generated by adding solution above the microfluidic device so that it would flow over the exit port due to gravity (See S2). This flow was added by drawing $3 \mathrm{~mL}$ of solution from the Petri-dish into a $3 \mathrm{~mL}$ syringe and manually dropping the solution onto the top of the microfluidic device. The pool of solution was positioned so that it would flow over the surface of the device and out over the exit of the channel in the same direction as the flow out of the device. The speed that the solution was added was changed to obtain situations where the drops would be travelling slower in the device than the surrounding solution. This involved dispensing the entire syringe over approximately 10 seconds. 


\section{Results and Discussion \\ Interfacial Tension}

By measuring interfacial tension with concentration, it is possible to observe the concentration regions where polymer-surfactant complexes are formed based on adsorption at the oil-water interface. The values of $T_{1}$ and $T_{2}$ can vary with molecular weight and length of the polymer chain, thus it was important to measure the specific characteristics for the system used (91). The interfacial tension for tetradecane in pure water was measured as $46.4 \mathrm{mN} / \mathrm{m}$ and tetradecane with $0.5 \%$ PVP was measured as $33 \mathrm{mN} / \mathrm{m}$, indicating that PVP molecules are absorbed at the oil-water interface in the absence of surfactant.

Figure 3 shows the variation of interfacial tension with SDS concentration at the oil-water interface for tetradecane with SDS aqueous solutions with no PVP and 0.5\% PVP, respectively. The PVP with SDS curve demonstrates a shape similar to that of other polymer-surfactant complexes; an overall decreasing interfacial tension, with two clear points of discontinuity either side of the CMC of SDS (roughly $8 \mathrm{mM}$ ) and a final plateau equivalent to that of the SDS curve at an interfacial tension of 8 $\mathrm{mN} / \mathrm{m}$ (39). The points of discontinuity for the system are observed at roughly $2.5 \mathrm{mM}$ SDS and $30 \mathrm{mM}$ SDS, encompassing the region at which it is expected that polymer-surfactant complexes are formed and exist on the interface. PVP and SDS have previously been shown by Chari et al. using radiotracers to form two-dimensional aggregates similar in structure to those in the bulk phase at the air-water interface (62). Purcell et al. (34) furthered the investigation using neutron reflection to show the presence of both PVP and SDS at the interface between $T_{1}$ and $T_{2}$, demonstrating evidence of a surface interaction between PVP and SDS. Using these data and previous studies on the adsorption at the airwater interface, the concentration range $2.5-30 \mathrm{mM}$ SDS was selected for investigating the adhesion between drops using direct force measurements and microfluidics.

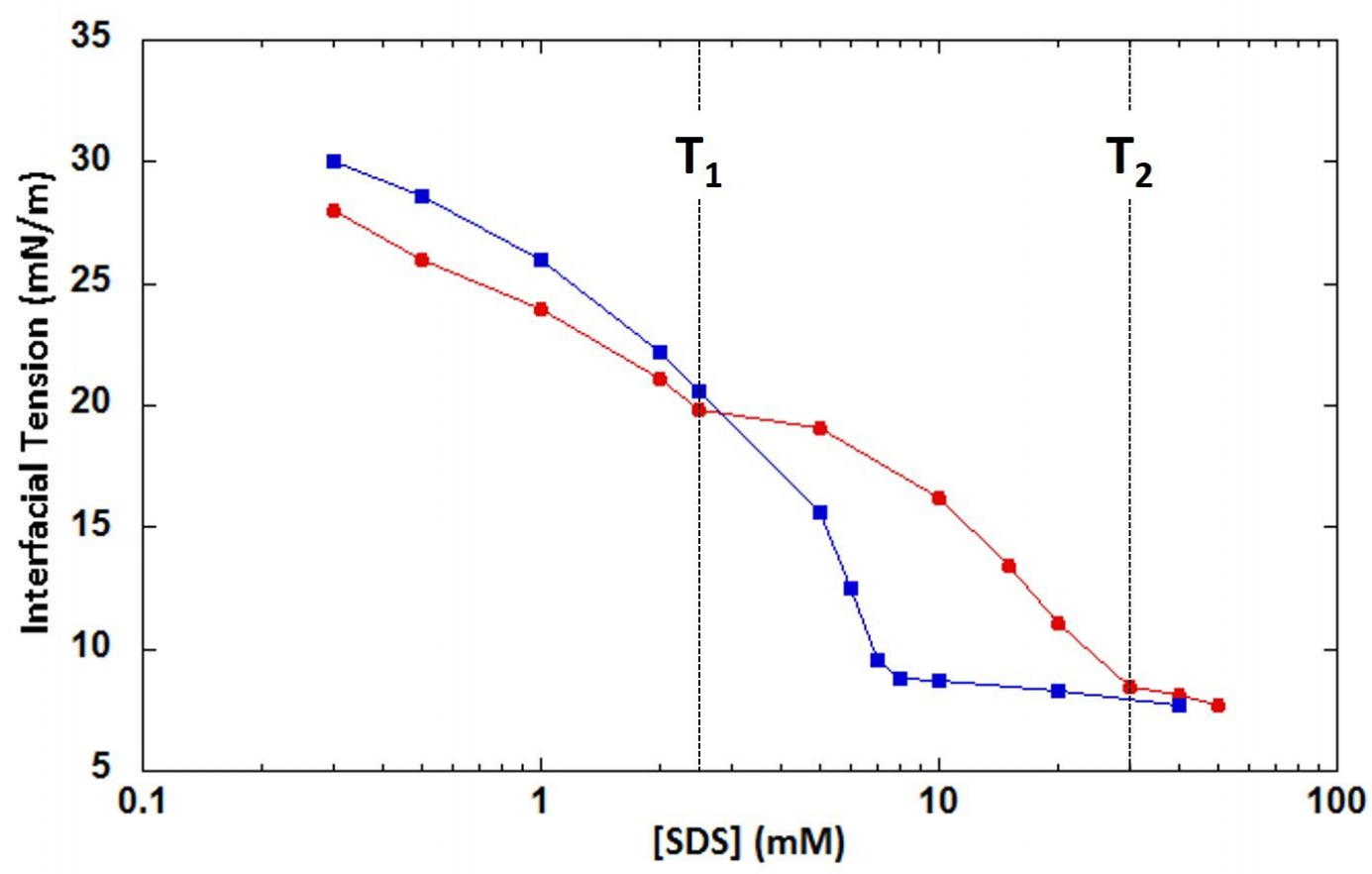

Figure 2 The interfacial tension between tetradecane SDS aqueous solutions (blue squares), and interfacial tension between tetradecane SDS aqueous solutions with 0.5\% PVP (red circles), across a range of SDS concentrations. The points $T_{1}$ and $T_{2}$ highlight the concentration region between which PVP/SDS complexes are expected to have formed and be located at the oil-water interface. 


\section{Direct Force Measurements}

\section{Rigid Surfaces}

AFM direct force measurements between a silica particle and glass slide in aqueous solution were conducted to quantify the attractive forces observed between oil drops in the presence of the PVP/SDS complex, but in the absence of interfacial deformation. Previous AFM studies between rigid interfaces in the presence of polyelectrolytes have accurately measured depletion forces and successfully fitted osmotic pressure models to the data (92-96).

Hydrophobic and hydrophilic surfaces were studied to determine the effective osmotic pressure for the SDS and PVP polymer-surfactant complexes across a range of SDS concentrations between $T_{1}$ and $T_{2}$. The AFM force curves measured between two hydrophilic silica surfaces (See S3(A)) exhibit increasing repulsion at close separation, consistent with the presence of a steric brush. The presence of a steric force obscures any attractive forces attributed between the surfaces, thus hydrophobically modified surfaces were used.

Force measurements between a hydrophobically modified $5 \mu \mathrm{m}$ silica particle and glass slide in solutions of $0.5 \%$ PVP and SDS concentrations of $2 \mathrm{mM}, 10 \mathrm{mM}$ and $30 \mathrm{mM}$ are shown in Figure 4(A). At $2 \mathrm{mM}$ SDS, below the CAC, the force curve exhibits a repulsive force attributed to electrical double layer repulsion, due to surfactant adsorption to the hydrophobic surfaces. However, at surfactant concentrations above the CAC an attractive force is observed, attributed to a depletion force where the magnitude of the attractive minima increases with increasing surfactant concentration. As additional surfactant is added to the system the number of surfactants bound to the polymer grows, effectively increasing the number of ions associated with the polymer. As discussed above, by treating the PVP/SDS complex as a pseudo-polyanion, this force behaviour is consistent with polyelectrolyte scaling theory, where the magnitude of the depletion force is primarily dependent on solution ionic strength and the ionic density of the polymer (97).
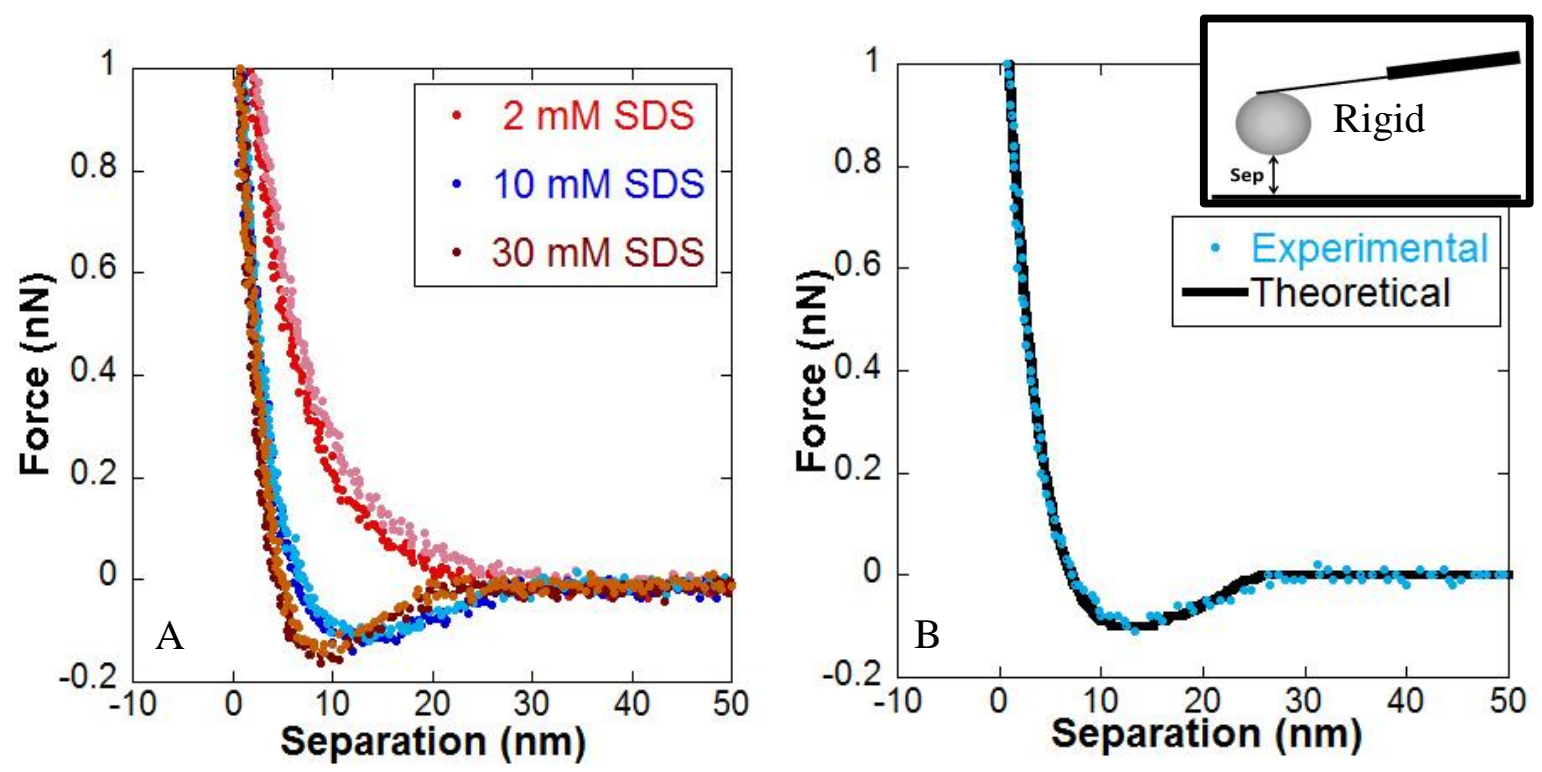

Figure $\mathbf{3}$ (A) The approach and retract AFM force curves between a silica particle and glass slide coated with gold and hydrophobically modified with decanethiol in an aqueous solutions of $0.5 \%$ PVP with $2 \mathrm{mM}$ SDS (red), $10 \mathrm{mM}$ SDS (blue) and $30 \mathrm{mM}$ SDS (brown) (B) A comparison between experimental data and the fitted model that describes the interaction force for this geometry in the presence of an EDL and depletion force for the case of an aqueous solution with $0.5 \%$ PVP and $10 \mathrm{mM}$ SDS. 
The surface force model to describe the EDL and depletion forces (equations 1-3) was fitted to the experimental data to determine the effective osmotic pressures, surface potential and depletion lengths for different SDS concentrations. In this system, there was no added electrolyte, thus the ionic strength was determined from the SDS concentration. Using the aggregation numbers and number of bound micelles per monomer, the dissociation of SDS molecules in bound micelles, free micelles or as free molecules in solution, were calculated via a mass balance in order to determine the ionic strength of the system (See supplementary material for further details).

As seen in Figure 4(B), a comparison between the output of the model and experimental data for $10 \mathrm{mM}$ SDS demonstrates the force data is well described as an EDL repulsion and depletion attraction. Based on the calculated ionic strength, the fitted depletion length and osmotic pressure are reported in Table 1 for SDS concentrations of $10 \mathrm{mM}, 15 \mathrm{mM}, 20 \mathrm{mM}$ and $30 \mathrm{mM}$ of SDS with $0.5 \%$ PVP. The depletion length generally decreases with increasing ionic strength. The length scale of the depletion layer is closely related to the characteristic length of the PVP/SDS complex in solution. As the number of SDS molecules increases within the system, the number of bound SDS molecules to the complex increases. This can increase the charge of the complex, but given the discussion of surfactant association above, this results in a weak dependence on concentration. However, the ionic strength also increases with the addition of surfactant, resulting in an increase in the amount of charge screening effects of the bound SDS molecules on the PVP, and thus reducing the effective characteristic length of the PVP/SDS pseudo-polyelectrolyte complex.

The surface potential shows a weak dependence on SDS concentration, which is not surprising given the partial dissociation of the SDS layer on a hydrophobic surface $(5,98)$. The fitted osmotic pressure is relatively constant within experimental error for 10 \& $15 \mathrm{mM} \mathrm{SDS}$ concentrations and then increases with SDS concentration at higher concentrations. In addition to fitting the osmotic pressure, by treating the PVP/SDS complex as a pseudo-polyelectrolyte complex or pseudo-polyanion, we can estimate an independent value for osmotic pressure using polyelectrolyte scaling theory defined by (99):

$$
\frac{\Pi_{\mathrm{OSM}}}{k T}=\frac{C^{2}}{4 A^{2} C_{s}+A C}+\frac{1}{\xi^{3}}
$$

where $A$ is the number of monomers per charge, $C$ is the polyelectrolyte monomer concentration and $C_{s}$ is the concentration of additional electrolyte. The characteristic length $\xi$ is related to the depletion layer thickness, equal to twice its length. Accounting for micelle disassociation, the number of monomers per charge can be calculated from the bound micelle aggregation numbers and number of PVP monomers per micelle. For the concentration range of 10-30 mM the number of monomers per charge was found to vary between 19.5 and 11.6. The concentration of additional electrolyte was determined in the SDS molecule mass balance (Supplementary Material). Combining these values with the calculated depletion length provides enough information to calculate an osmotic pressure directly from Eq. 4, providing a comparative value to those determined from the fitted model in Table 1 . The calculated values show a similar trend but are systemically higher than the values fit to the AFM data directly. The difference between these values is most likely due to polydispersity effects, unaccounted for in the scaling theory (97). Previous direct force measurements between bubbles or rigid surfaces using both AFM and total internal reflection microscopy measurements $(28,94,100)$ have observed a reduction in the measured osmotic pressure due to the polydispersity of the depletant for both charged and neutral depletants. In these instances, it was found that the larger size fraction of the 
depletant set the length scale of the depletion length, but the small size fraction of the depletant was not excluded from the gap, leading to a reduction in the osmotic pressure measured via force measures measurement compared to the osmotic pressure calculated based on scaling theory. These data and force model, describing the depletion force between rigid hydrophobic surfaces will be used in the analysis framework that accounts for interfacial deformation for the force data between two oil drops discussed below.

Table 1 Summary of the depletion length and osmotic pressure determined by fitting an EDL and depletion model to AFM force curve data between rigid hydrophobic surfaces as well as the Osmotic Pressure calculated via polyelectrolyte scaling theory.

\begin{tabular}{lllllll}
$\begin{array}{l}\text { SDS } \\
\text { Concentrati } \\
\text { on }(\mathbf{m M})\end{array}$ & $\begin{array}{l}\text { lonic } \\
\text { Strength } \\
(\mathbf{m M})\end{array}$ & $\begin{array}{l}\text { Calculated } \\
\text { Debye } \\
\text { Length } \\
(\mathbf{n m})\end{array}$ & $\begin{array}{l}\text { Fitted } \\
\text { Surface } \\
\text { Potential } \\
(\mathbf{m V})\end{array}$ & $\begin{array}{l}\text { Fitted } \\
\text { Depletion } \\
\text { Length } \\
\mathbf{( n m )}\end{array}$ & $\begin{array}{l}\text { Fitted } \\
\text { Osmotic } \\
\text { Pressure } \\
(\mathbf{P a})\end{array}$ & $\begin{array}{l}\text { Osmotic } \\
\text { Pressure } \\
(\mathbf{P a}) \\
\text { (Scaling } \\
\text { Theory) }\end{array}$ \\
\hline $\mathbf{1 0}$ & $7.2 \pm 0.3$ & 3.6 & $-15 \pm 2$ & $12.8 \pm 0.9$ & $667 \pm 65$ & $879 \pm 202$ \\
\hline $\mathbf{1 5}$ & $9.1 \pm 0.7$ & 3.2 & $-15 \pm 2$ & $11.5 \pm 0.8$ & $572 \pm 51$ & $1017 \pm 285$ \\
\hline $\mathbf{2 0}$ & $11.7 \pm 0.5$ & 2.8 & $-15 \pm 2$ & $11.4 \pm 0.6$ & $738 \pm 63$ & $1141 \pm 280$ \\
\hline $\mathbf{3 0}$ & 13.94 & 2.6 & $-16 \pm 2$ & $9.7 \pm 1.0$ & $1117 \pm 109$ & $1397 \pm 75$ \\
\hline
\end{tabular}




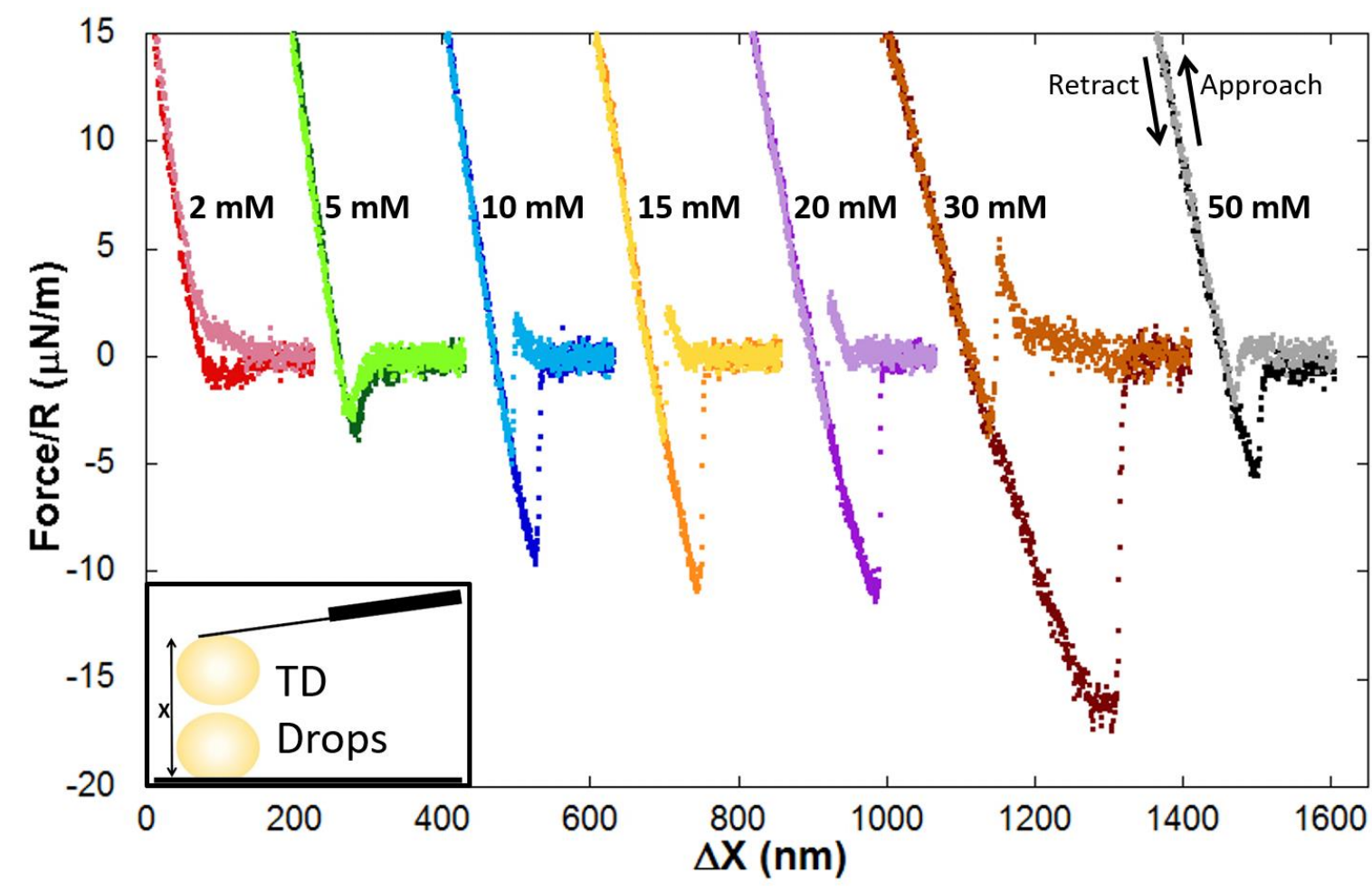

Figure 4 The interaction force between two tetradecane drops in aqueous solution measured by AFM normalised by average drop radius versus $\Delta \mathrm{X}$ in the presence of $0.5 \% \mathrm{PVP}$ and a range of SDS concentrations. These data are translated on a relative $\Delta \mathrm{X}$ scale for ease of viewing. The SDS concentration spanned below $\mathrm{T}_{1}$ at $2 \mathrm{mM}$ SDS (light red approach, dark red retraction), above $\mathrm{T}_{1}$ at $5 \mathrm{mM}$ SDS (light green approach, dark green retraction), $10 \mathrm{mM}$ SDS (light blue approach, dark blue retraction), $15 \mathrm{mM}$ SDS (yellow approach, orange retraction), $20 \mathrm{mM}$ SDS (light purple approach, dark purple retraction), and $30 \mathrm{mM}$ SDS (light brown approach, dark brown retract) and above $T_{2}$ at $50 \mathrm{mM}$ SDS (grey approach, black retract). These curves were taken with a piezo drive velocity of $100 \mathrm{~nm} / \mathrm{s}$.

Force curves for the interactions between two tetradecane drops in $0.5 \mathrm{wt} \%$ PVP over a range of SDS concentrations is shown in Figure 5 . To facilitate a qualitative comparison between drop pairs, the force has been normalised by the average drop radius to account for variations in drop size across different measurements. These measurements were performed at piezo drive velocities of $100 \mathrm{~nm} / \mathrm{s}$ as hydrodynamic effects are expected at higher piezo drive velocities, which is explored further in the following section.

The force curve for the $0.5 \mathrm{wt} \%$ PVP and $2 \mathrm{mM}$ SDS solution exhibits a small hysteresis between the approach and retract curves with a small repulsive force at close range attributed to EDL forces. This behaviour is consistent with that observed in a control measurement of $0.5 \mathrm{wt} \%$ PVP without SDS and is not attributed to a depletion force from the PVP/SDS complex (Supplementary Material). When the concentration of SDS is increased to $5 \mathrm{mM}$, the force behaviour changes to an attractive profile on both approach and retract. At close separation, the attractive region exhibits a small hysteresis consistent with the presence of an attractive surface force, but the drops do not coalesce $(84,87)$. 
The $10 \mathrm{mM}$ solution exhibits significant hysteresis between approach and retraction without drop coalescence. On approach there is a small but significant repulsion followed by a jump in to an attractive force region. The retraction is defined by a significant attractive force followed by a jump off as the drops move apart without coalescence. The jump in behaviour in attractive force fields from depletion or structural force between drops and bubbles has been observed previously, for polyelectrolytes, micelles, nanoparticles, or microemulsion depletants, and modelled in some detail $(31,52)$. Thus, the presence of the jumps on approach and retract and the increasing hysteresis is attributed to the drop deformation caused by the equilibrium disjoining pressure. The 15 to $30 \mathrm{mM}$ SDS solutions exhibit force behaviour very similar to the $10 \mathrm{mM}$ case. However, there are two significant changes observed in the force profiles with increasing surfactant concentration. Firstly, there is an increase in the magnitude of the repulsion on approach, which is noticeably larger at a concentration of $30 \mathrm{mM}$. Moreover, there is a gradual increase in the attractive force seen in the retract curve until a large jump in magnitude at $30 \mathrm{mM}$ SDS. At $50 \mathrm{mM}$ there is no longer any evidence of a repulsive force on approach, demonstrating behaviour more similar to that seen at $5 \mathrm{mM} \mathrm{SDS}$. A large reduction in the attractive force compared to the $10-30 \mathrm{mM}$ concentrations is also measured.

Qualitatively, the changes in the attractive force are attributed to changes in the PVP/SDS complex mediating a depletion force, whereas the changes in the repulsive force are associated with the adsorption and then desorption of the PVP/SDS complex from the oil-water interface with increasing SDS concentration. As discussed above, at $2 \mathrm{mM}$ SDS, the repulsive force is attributed to the surfactant, similar to the control measurements. From $5 \mathrm{mM}$ to $30 \mathrm{Mm}$, depletion interactions are present and increase in size with added surfactant. Similar to the rigid force measurements at these concentrations, SDS and PVP aggregate in the bulk resulting in a complex that effectively acts as a pseudo-polyelectrolyte leading to a depletion force between the drops. The increase in depletion force is due to the accumulation of adsorbed SDS molecules to the PVP, resulting in an increasing counter ion density of the pseudo-polyelectrolyte. At $50 \mathrm{mM}$, depletion is still prevalent although it is greatly reduced in magnitude. Despite the fact that SDS is still adsorbed to the PVP, the polymer is now expected to be completely saturated such that any additional SDS will act effectively as added electrolyte, increasing the ionic strength and ultimately reducing the osmotic pressure via screening of the repulsive SDS/PVP complex intra- and inter-actions, and thus the depletion force. To further support this analysis, and explain the origins of the hysteresis in the approach and retract force curves, a comparison of these force data to the SRLY model is presented below.

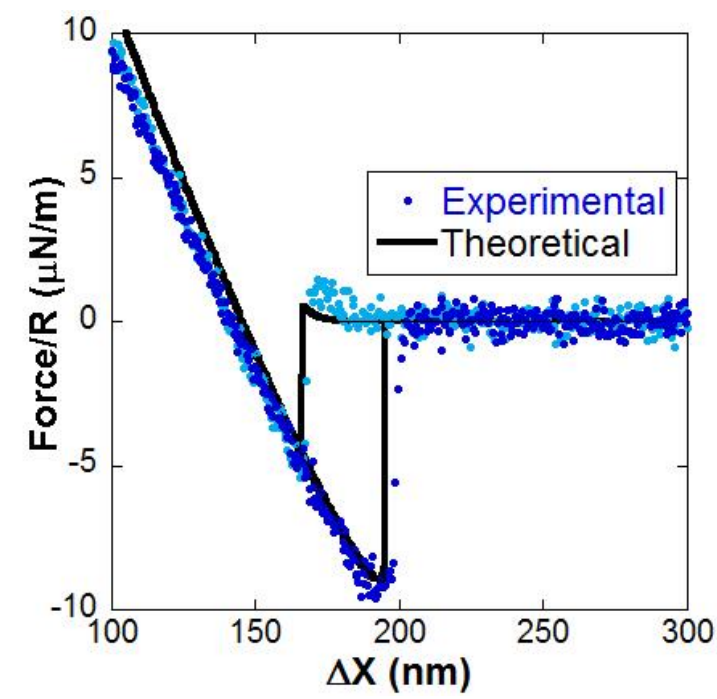

Figure 5 Force divided by average drop radius versus $\Delta \mathrm{X}$ for the interaction between two tetradecane drops comparing experimental data and theoretical output for $0.5 \mathrm{wt} \%$ PVP with 10mM SDS. 
These AFM force measurements were analysed using the SRYL model outlined above, assuming no significant hydrodynamic drainage effects, but accounting for the presence of EDL and depletion forces, as well as the deformation of the interface, in order to determine the separation between the drops. The osmotic pressure, depletion length, ionic strength and Debye length were defined using the values determined from the rigid force fit measurements such that the only adjustable parameter in the model was the surface potential of the drops. Figure 6 shows a comparison of the modelling results to the experimental results for an SDS concentration of $10 \mathrm{mM}$. The agreement between the model and the experiment supports the use of the depletion parameters and ionic strength calculations determined in the rigid measurements. Independently measured values for the drop radii, interfacial tension, drop-substrate and drop-cantilever contact angles, and cantilever spring constant were also used. A list of parameters used for the analysis in Figure 6 and their associated uncertainties are given in the SI.

For the $10 \mathrm{mM}$ data in Figure 6, there is an under prediction in the model of the repulsion on approach, suggesting that the EDL forces are not sufficient to describe the entire repulsive force. The EDL force does not account for a contribution from a steric force due to adsorbed PVP/SDS complex. Based on the interfacial tension measurements in Figure 3, and previous neutron scattering of the PVP/SDS complexes adsorbed at an air water interface (34), adsorption of the PVP/SDS complex is expected at the drop interface. The steady growth of repulsion in the approach force curves for SDS concentrations from 10 to $30 \mathrm{mM}$ in Figure 5 is attributed to the increase of SDS within the complex. Similar to the bulk behaviour, as the amount of SDS increases in the complex, the number of bound micelles and the aggregation number of these micelles grows, creating more intra- and inter-complex repulsion within the adsorbed complex layer. This leads to the layer swelling, resulting in an electro-steric repulsion between the drops at close separations as seen in Figure 6. Thus, the details that would need to be captured to include an electro-steric contribution in the surface forces go well beyond a simple steric model and were not included in the SRYL model. At $50 \mathrm{mM}$ there is no longer any evidence of a repulsive force. At this surfactant concentration the solution is above $T_{2}$, where the polymer is completely saturated with surfactant, and it is expected that the complex has desorbed from the interface. The attractive well observed during retract is well described by the model (Figure 6). The magnitude of the adhesion corresponds to attractive depletion but is also affected by the deformation of the drops at close separation. Previous studies of depletion and structural forces between drops using simpler depletants have shown that the interfacial deformation makes the probe more sensitive to these attractive interactions than a rigid sphere of the same radius (29-32). In addition, the interfacial deformation is the origin of the large hysteresis between the approach and retract, as the drops flatten by the point of maximum force, thus there is a larger interaction area when the drops are separated and sampling the attractive depletion force. At 30mM SDS, there is a significant increase in both the electro-steric repulsion and the adhesion due to the onset of desorption and swelling of the complex from the interface $(101,102)$.

\section{Dynamic}

A comparison of the dynamic interactions between two tetradecane drops in $10 \mathrm{mM}$ SDS and $0.5 \mathrm{wt} \%$ PVP over nominal velocities of 1-10 $\mu \mathrm{m}$ and a maximum force of approximately $2 \mathrm{nN}$ is shown in Figure 7. The increase in the velocity dependent hysteresis is attributed to hydrodynamic drainage effects. This is consistent with previous studies using drop radii between 10 and $100 \mu \mathrm{m}$, where strong coupling between hydrodynamic and surface force interactions is observed $(1,5,7)$. Similar to earlier studies of drops/bubbles in the presence of depletion and/or structural forces -- with either surfactants or polyelectrolytes (28-30) -- it is clear that as the velocity increases, the depletion behaviour apparent in the equilibrium measurements shown in Figure 6 is overwhelmed by 
hydrodynamic effects. The increasing effect of hydrodynamic drainage is evident in the increasing hysteresis, which eventually softens the well-defined sharp features from the repulsion and attraction to a smooth transition as a result of the large hydrodynamic pressure within the film. The current work differs to previous studies, where we have used the SRYL model to describe these force data, shown in Figure 7, using the osmotic pressure, surface potential and characteristic depletion length extracted from fitting the equilibrium measurements given in Table 1 or in the SI, Table S3. Noticeably, there is a strong agreement between the model and the experimental results over the range of velocities. However, although the approach curve is tracked well by the model, there is a visible under-prediction of the attractive force on the retract curve. A study by Manor et al. explored the effects of film drainage through a steric polymer brush at the interface between two decane drops (103). They highlighted that the film drainage through an adsorbed polymer layer should not be treated as a simple interface, showing through continuum modelling that the film drainage between drops is sensitive to flow through the brush. Thus, the deviation between the model and measurement in Figure 7 is consistent with the presence of a steric layer, where a portion of the hydrodynamic drainage occurs within the absorbed PS complex layer on the drops. The dynamic results highlight the importance of controlling hydrodynamic drainage effects when measuring the interfacial forces between drops in polymer-surfactant systems. Thus, in drop collisions, to accurately observe the equilibrium surface forces, such as adhesion between drops in a microfluidic device it is therefore necessary to mitigate hydrodynamic interference.

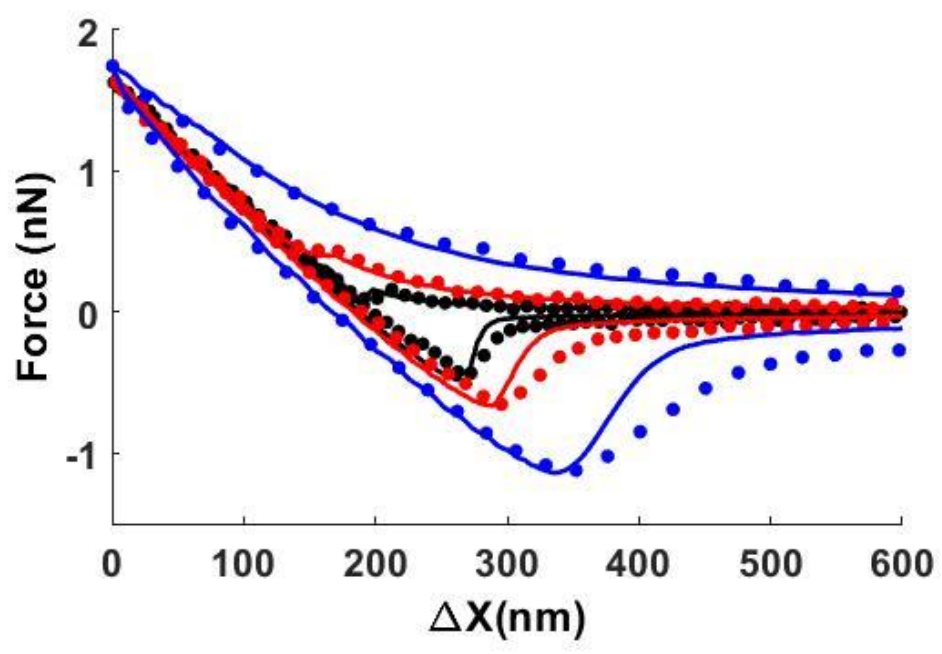

Figure 6 Comparison of the force vs displacement between two tetradecane drops in $10 \mathrm{mM}$ of SDS and $0.5 \%$ PVP at velocities of (black) $1 \mu \mathrm{m} / \mathrm{s}$, (red) $3 \mu \mathrm{m} / \mathrm{s}$ and (blue) $10 \mu \mathrm{m} / \mathrm{s}$. The graph shows both the experimental results (dots) and the theoretical prediction of the StokesReynolds-Young-Laplace model (Lines). 


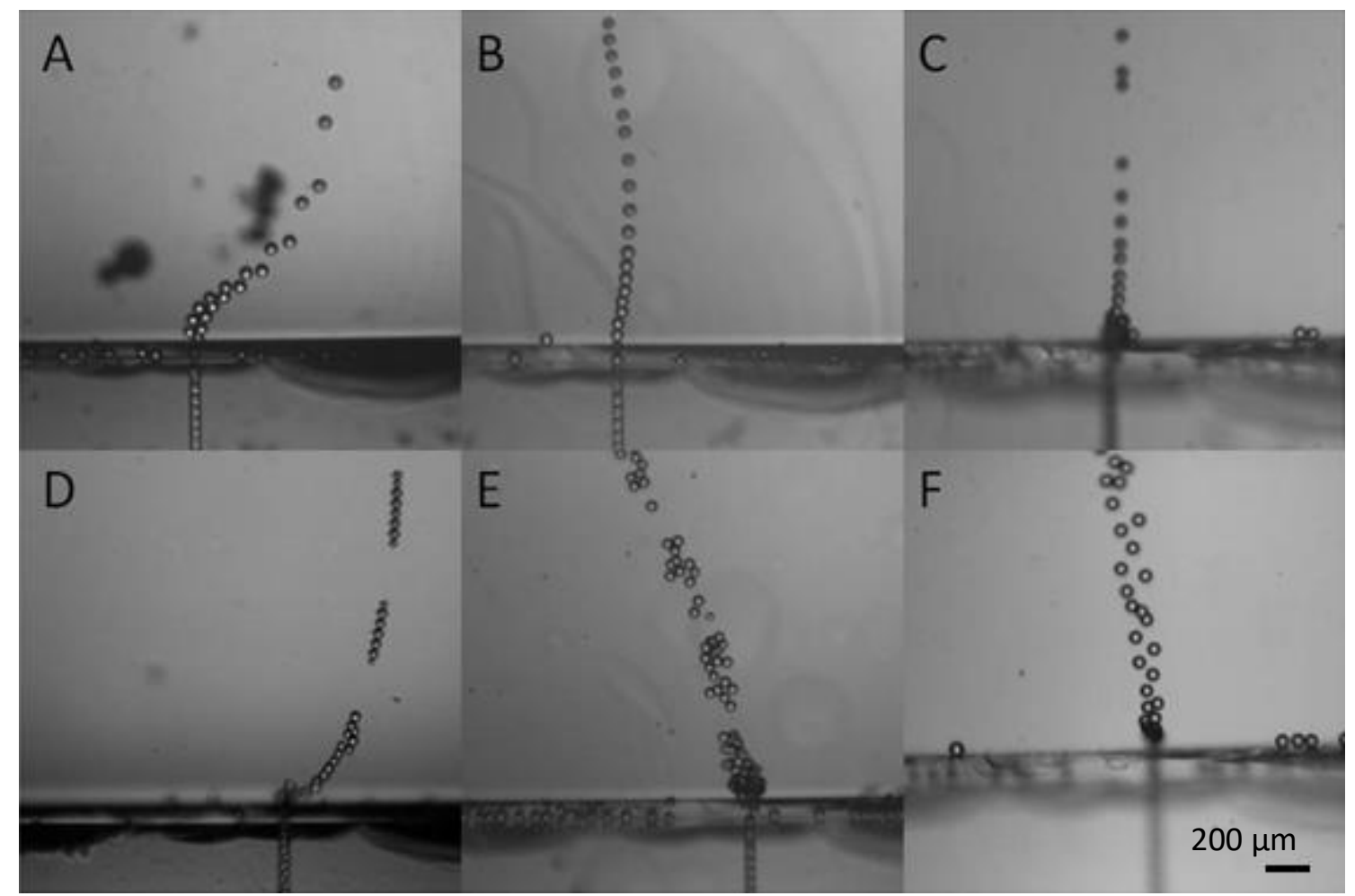

Figure 7 Representative images of the breakup of tetradecane drop chains at the exit of the microfluidic device due to external flow. Solutions used are a) $10 \mathrm{mM} \mathrm{SDS}$, b) $2 \mathrm{mM}$ SDS and 0.5\%PVP, c) $5 \mathrm{mM}$ SDS and 0.5\%PVP, d) $10 \mathrm{mM}$ SDS and 0.5\%PVP, e) $15 \mathrm{mM}$ SDS and 0.5\%PVP, and f) $20 \mathrm{mM}$ SDS and 0.5\%PVP. Flowrates were set on the fluid pumps as $\mathrm{Q}_{c}=30 \mu \mathrm{L} / \mathrm{hr}$, $\mathrm{Q}_{D}=10 \mu \mathrm{L} / \mathrm{hr}$, and $\mathrm{Q}_{\mathrm{s}}=11 \mu \mathrm{L} / \mathrm{hr}$, resulting in $\mathrm{Q}_{0}=18 \mu \mathrm{L} / \mathrm{hr}$, giving drop with an equivalent spherical radius of $\sim 28 \mu \mathrm{m}$ and a drop velocity of $\sim 2 \mathrm{~mm} / \mathrm{s}$.

\section{Microfluidics: Equilibrium Interactions}

A microfluidic device was developed, schematically shown in Figure 1 and in more detail in the supplemental information, to probe the attractive or adhesive interactions between drops. The goal of this work was to form chain-like structures of drops held together via equilibrium surfaces forces and then exit a microfluidic channel into a bulk, sweeping flow. In any drop based microfluidic device where drop collisions occur, hydrodynamic drainage forces occur, and as discussed above in the study by Anna and co-workers (18), often dictate the outcomes of the collisions masking surface force effects. As shown in Figure 7, even a relative velocity difference of 5 microns/s is sufficient for hydrodynamic drainage effects to mask the depletion forces encountered from the PVP/SDS complex. We developed a device where the drops come into close contact via the introduction of side channels, similar to those used in drop coalescence studies by Gunes et al. (17), enabling the removal of excess continuous phase (see movie, supplemental information, SV1), driving the drops into close separation with the same relative velocity, but without coalescence. The drops continue in this geometry moving through the device (see movie, supplemental information, SV2-6), and exit the device where the persistence of the drop chain is expected to correlate with the attractive interactions between the drops.

The formation of drop chains in solutions covering a range of SDS concentrations in the absence and presence of $0.5 \% \mathrm{w} / \mathrm{w}$ PVP was studied using the microfluidic device, where frames from movies of the operation of the device are shown in Figure 8. A control measurement for $10 \mathrm{mM}$ SDS in Figure 8A shows the drops momentarily bunch near the exit of the microfluidic device before breaking apart into individual drops downstream. This is understandable as a small region near the microfluidic device 
is likely protected from the external flow due to the thickness of the device allowing the drops to bunch up in this area and push against each other. Once the drops move past this area the external flow is much stronger and the drops break apart due to the lack of any adhesive or strong attractive forces.

For the $2 \mathrm{mM}$ SDS 0.5\% w/w PVP solution shown in Figure 8B the drops maintain contact upon exiting the microfluidic device and form a single line of touching drops in the direction of fluid flow unlike the control measurement that separated immediately. Once the drops move into the region of significant external fluid velocity they break apart immediately into individual drops without exception. This is expected from the force curves as no strong attractive forces were observed for this SDS concentration. The $5 \mathrm{mM}$ SDS $0.5 \% \mathrm{w} / \mathrm{w}$ PVP solution shown in Figure $8 \mathrm{C}$ behaves in a similar fashion, where very occasionally double or triple drop chains persist moving into the external flow field. This is attributed to a possible drop adhesion consistent with the increasing attraction observed in the AFM data at this concentration. It is important to note that in the region outside of the device, forces from both drag due to fluid motion and buoyancy act on the drops opposing the adhesive force between them. The approximate nature of the sweeping flow is difficult to quantify, but the buoyancy force, based on the drop radii is of the order of $1 \mathrm{nN}$.

The $10 \mathrm{mM}$ SDS 0.5\% w/w PVP solution shown in Figure 8D demonstrates significantly more drop aggregation. In this solution the drops would exit the microfluidic device and maintain contact as they moved into the stronger flow field. Unlike the previous solutions the drops moved significantly farther into bulk solution with neighbouring drops moving over each other while maintaining contact to align in the flow. The drops would then break off into stable groups of roughly between 6 and 10 drops depending on the flowrate. This implies that the adhesive forces between each drop are strong enough such that the drag and buoyancy force on each drop is not enough to break the chain into individual drops.

The $15 \mathrm{mM}$ SDS $0.5 \% \mathrm{w} / \mathrm{w}$ PVP solution shown in figure 8E exhibits behaviour that is less adhesive than the $10 \mathrm{mM}$ SDS solution but still demonstrates attractive forces between drops. The drops do not form the desired chains, instead forming loose clumps at the exit of the device that break apart into individual drops after a short time. The $20 \mathrm{mM}$ SDS $0.5 \% \mathrm{w} / \mathrm{w}$ PVP solution shown in figure $8 \mathrm{~F}$ demonstrates no signs of adhesive forces between the drops. The drops in this solution break apart very quickly upon leaving the microfluidic device and spread out into two alternating streams of drops.

Each solution was run within the device a number of times with the rate that the added solution was dispensed intentionally varied to try and ensure that the drops experienced a variety of different flow fields so that the exact conditions needed to just break the drops apart could be observed. If the flow was too gentle the drops would pool near the channel exit but if the flow was too strong the drops would break apart rapidly. The presented results are indicative of the strongest aggregation between drops that was observed for each solution.

An important factor to consider regarding the adhesive forces between the drops is the length of time that the drop spends within the microfluidic channel. The observation of adhesive forces is dependent on the adsorption of the PVP/SDS complex onto the drop interface. This is influenced by both drop lifetime and surfactant concentration. Depending on the flow rates, the time between drop generation and collision will vary but is normally on the scale of seconds. Post drop collision, the time the drops maintain close proximity in the device, is again on the scale of seconds (up to 10 seconds). Describing the transport and adsorption process in this device quantitatively will be a focus of subsequent work, however we note here that the adsorption time scales within the channel are expected to be faster than bulk measurements such as dynamic pendant drop tensiometry (104). 


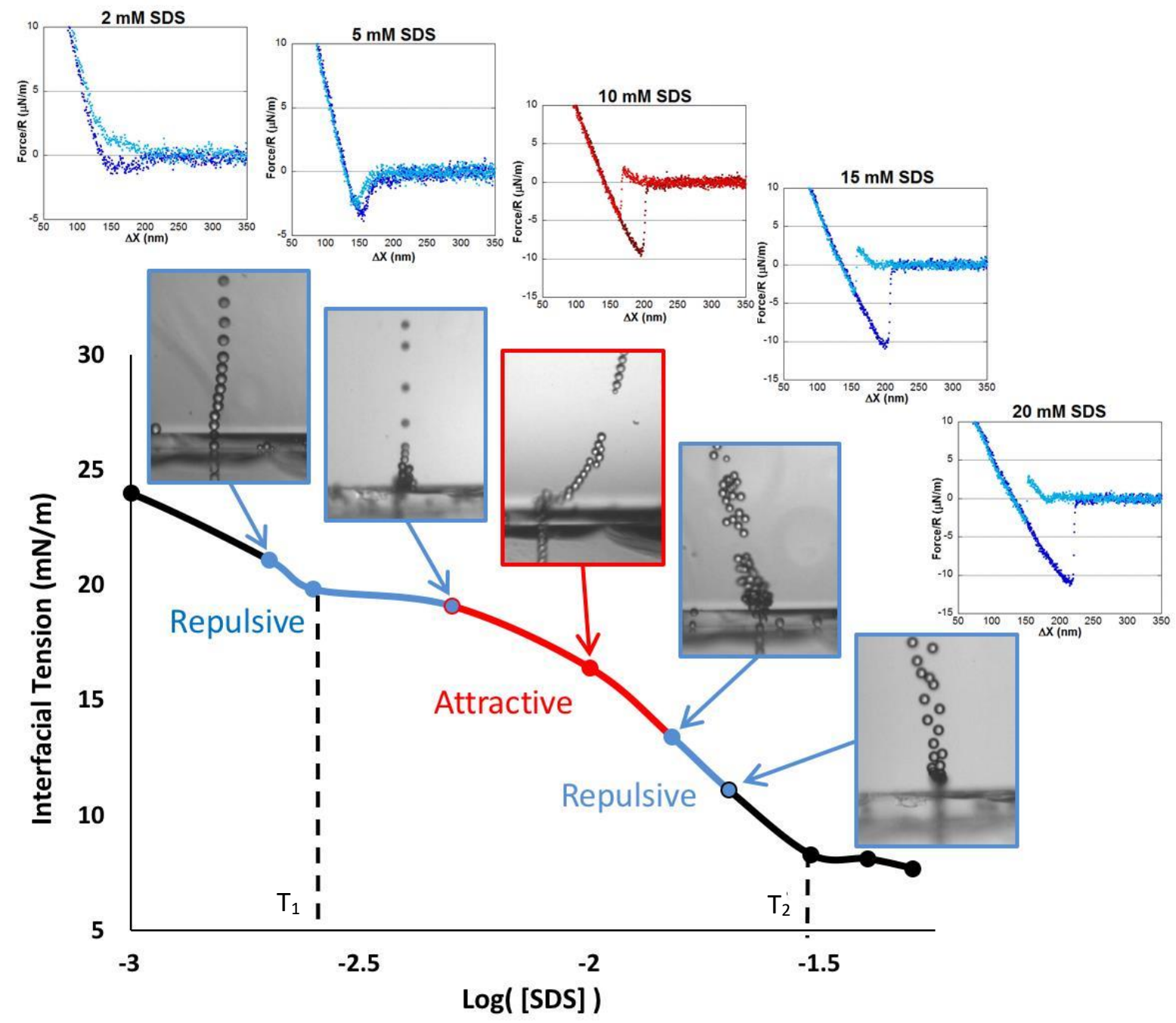

Figure $8 \mathrm{~A}$ diagram comparing the results from the interfacial tension measurements (defining the $T_{1}$ and $T_{2}$ region), microfluidic observations and drop-drop AFM measurements. The results highlighted in blue illustrate the data that returned net repulsive behaviour, while the red demonstrate the data that returned a net attractive behaviour.

\section{Linking AFM and Microfluidic Observations}

Figure 9 shows an overview of the microfluidic observations and direct force measurements with respect to their corresponding concentration on the interfacial tension isotherm. Our original hypothesis was that drop adhesion would occur between the $T_{1}$ and $T_{2}$ concentrations (at $2.5 \mathrm{mM}$ and $30 \mathrm{mM}$ respectively). In the AFM force measurements, large adhesive wells were observed between these concentrations. However, drop chaining was only observed at $10 \mathrm{mM}$ SDS in the microfluidic experiments with some drop adhesion observed at $5 \mathrm{mM}$ and $15 \mathrm{mM}$. In contrast to the AFM measurements, the microfluidic observations give insight into the stability of the drop chains. Upon exiting the channel into bulk solution, the stability of the drop chains is governed by a balance between drag, buoyancy, and the adhesive forces between the drops. If the adhesive forces between the drops are too weak, the drop chains break up upon exiting the channel. Further, for the most attractive surfactant concentrations, drops will detach from the drop chain until the external force acting on the chain is low enough such that a stable length is reached. 
The comparison of the AFM results to the microfluidics also demonstrates that the microfluidics is primarily path dependent, such that the attractive forces at close separation can only be experienced when the Laplace pressure is high enough to overcome the repulsive barrier encountered as the drops approach each other. In the microfluidic device drops move together due to the removal of continuous phase, and as a consequence the collision is not as controlled as a collision measured using AFM, where one drop is driven towards another by a prescribed distance and velocity. Thus, it is reasonable to expect that a drop collision in the microfluidic device may not be of sufficient force to overcome the repulsive barrier upon approach. Berry and Dagastine (1) defined a drop equilibrium stability criterion such that if the Laplace pressure is less than the repulsive force, the film between the drops will not drain enough to reach attractive forces capable of inducing coalescence or stable adhesion. It is therefore likely that as the steric repulsion gets bigger at $20 \mathrm{mM}$, despite an obviously larger attraction present in the force curve, the necessary force of collision required to overcome repulsion as the drops approach each other is not achieved and thus drop chains are not observed. Therefore, the longest stable drop chain correlates with the SDS concentrations in the AFM measurements ( $\sim 5$ $15 \mathrm{mM}$ ) when the repulsive force on approach is low enough, and the attractive force at close separation is high enough, to keep the drops in adhesive contact. This suggests that the droplet interactions in the microfluidic channel can identify at what SDS concentrations the attractive force dominates for that specific drop size. Thus, for this technique to be broadly used to probe surface forces between drops, it is necessary to produce drops with a range of sizes in order to sample a range of Laplace pressures. Smaller drops with higher Laplace pressures can potentially push through the repulsive barrier, move closer together and thus be able to encounter the attractive forces also present at higher concentrations.

This study demonstrates the clear potential for using microfluidic devices to investigate surface forces, specifically net adhesive interactions. Clearly there are many areas that could benefit from greater control and more accurate measurements; in particular the flowrate of external fluid near the observation region. The variation in internal flow rates from the fluid syringe pumps also limited the ability to model the drop breakup or adsorption kinetics in this instance. An additional concern is understanding the impact of wall effects on the observed behaviour. It is important to note in this work, and the work by Shen et al., where they formed drop aggregates for photo-polymerisation, the structure was formed and persisted outside of a confined channel (76). In Shen's work, a large expansion with a controlled co-flow, a desirable attribute in our next design, was used. In our work, the drop chains were observed in a gentle co-flow region completely outside of the device. Effects from internal circulation of the drop are often minimized by high coverage of surface-active materials, low flow rates and when the continuous phase viscosity is much less than the drop phase, as it is in this instance (105). 


\section{Conclusion}

Through interfacial tension measurements, direct force measurements and microfluidic observations we have provided a detailed investigation into drop-drop interactions in a sodium dodecyl sulphate (SDS) and poly(vinylpyrrolidone) (PVP) solution. Interfacial tension measurements were taken between solutions of varying SDS concentration and 0.5\% PVP and tetradecane. The resulting curve demonstrated a profile that is consistent with previous observations of polymer-surfactant surface tensions and determined the existence of polymer-surfactant complex formation and interfacial adsorption within the concentration range of $2.5 \mathrm{mM}$ SDS to $30 \mathrm{mM}$ SDS. Rigid force measurements showed that depletion forces dominate the interactions between two hydrophobic surfaces in these systems. The magnitude of the attractive well exhibited surfactant concentration dependence, increasing with increasing amounts of SDS. These data were quantitively described with an electrical double layer (EDL) and depletion model to determine depletion length and osmotic pressure. In addition, by treating the PVP/SDS complex as an effective polyanion, the measured osmotic pressure was compared favourably to calculated values using polyelectrolyte scaling theory. Interaction forces between two deformable oil drops were measured over the same PVP and SDS concentrations and displayed similar depletion behaviour. At $2 \mathrm{mM}$, prior to polymer-surfactant (PS) complex formation, there was no evidence of depletion. Within the SDS concentrations that complex formation is expected $(2.5-30 \mathrm{mM})$, an attractive region on approach was observed followed by an attractive well on retract. The magnitude of attraction was shown to increase with increasing SDS concentration. From $10 \mathrm{mM}$ a repulsion on approach prior to the close range attractive force is also seen, peaking in magnitude at $30 \mathrm{mM}$. By $50 \mathrm{mM}$, where the polymer is saturated with surfactant, the behaviour changes, showing no repulsion and a largely reduced attraction. A model used to describe deformable systems, accounting for the same depletion and EDL forces, used the parameters fit from the rigid surface analysis, including the depletion length and osmotic pressure, and showed reasonable agreement with the force data. A small under prediction by the model of the repulsive force on the approach curve suggests the existence of an additional steric force from the presence of adsorbed PS complexes at the interface. At velocities above $500 \mathrm{~nm} / \mathrm{s}$, dynamic forces, caused by film drainage between the drops, dominated the force data, such that it was no longer possible to observe the depletion or EDL force features in the measurements. A microfluidic device that minimized the influence of hydrodynamic drainage effects between drops was developed to allow for the observation of surface forces between drops. The microfluidic device was able to independently demonstrate the existence of attractive forces between the drops in a mixture of SDS and PVP and also create short drop chains held together by surface forces. Although attractive interactions were observed in the atomic force microscopy (AFM) measurements between $2.5 \mathrm{mM}$ and $30 \mathrm{mM}$ SDS for a PVP concentration of $0.5 \% \mathrm{w} / \mathrm{w}$, solutions at the border of this concentration range did form drop chains. Of the concentrations tested the $10 \mathrm{mM}$ SDS concentration successfully showed significant drop adhesion and chain formation with some adhesion between drops at an SDS concentration of 15 $\mathrm{mM}$. The lower SDS concentrations are assumed to lack sufficient adhesive forces to hold the drops together, whereas the large repulsive force seen in the AFM data at higher SDS concentrations prevents the drops entering the attractive force region required to promote drop chain formation.

The evidence presented here provides a detailed insight into the force behaviour between drops and how the PVP/SDS complex in the bulk and at the interface can mediate the forces between both rigid and deformable interfaces. This conclusion supports the findings of both Chari and Purcell $(34,62)$, however also provides greater insight into the characterisation of SDS and PVP between and on oilwater interfaces. Correlating the AFM and microfluidic results suggest that attractive forces observed in the AFM lead to the formation of drop chains when the repulsive force on approach is low enough to allow the drops to move into close separation, and when the attractive force is sufficient to hold 
the drops in a secondary attractive minimum. Increasing the Laplace pressure by generating smaller drops could allow for a wider range of adhesion to be observed, potentially enabling the development of a matrix of conditions for which adhesive emulsions can be formed across different system concentrations and drop sizes. Further developing this device could provide a high throughput technique for screening through a range of systems with ease, aiding formulation development where adhesive emulsions are important in areas such as pharmaceuticals, coatings and personal care products.

\section{Acknowledgements}

This work was performed in part at the Melbourne Centre for Nanofabrication (MCN) in the Victorian Node of the Australian National Fabrication Facility (ANFF). This work was performed in part at the South Australian node of the Australian National Fabrication Facility, a company established under the National Collaborative Research Infrastructure Strategy to provide nano and micro-fabrication facilities for Australia's researchers. This work was performed in part in the Materials Characterisation and Fabrication Platform (MCFP) at the University of Melbourne. We thank the ARC for funding and the PFPC for providing infrastructure support. 


\section{References}

1. Berry JD, Dagastine RR. Mapping coalescence of micron-sized drops and bubbles. Journal of Colloid and Interface Science. 2017;487(Supplement C):513-22.

2. Tabor RF, Chan DYC, Grieser F, Dagastine RR. Anomalous Stability of Carbon Dioxide in pHControlled Bubble Coalescence. Angewandte Chemie. 2011;123(15):3516-8.

3. Vakarelski IU, Manica R, Tang X, O'Shea SJ, Stevens GW, Grieser F, et al. Dynamic interactions between microbubbles in water. Proceedings of the National Academy of Sciences. 2010;107(25):11177.

4. Gunning AP, Mackie AR, Wilde PJ, Morris VJ. Atomic Force Microscopy of Emulsion Droplets: Probing Droplet-Droplet Interactions. Langmuir. 2004;20(1):116-22.

5. Dagastine RR, Manica R, Carnie SL, Chan DYC, Stevens GW, Grieser F. Dynamic Forces Between Two Deformable Oil Droplets in Water. Science (Washington, DC, United States). 2006;313(5784):2103.

6. Shi C, Cui X, Xie L, Liu Q, Chan DYC, Israelachvili JN, et al. Measuring Forces and Spatiotemporal Evolution of Thin Water Films between an Air Bubble and Solid Surfaces of Different Hydrophobicity. ACS Nano. 2015;9(1):95-104.

7. Tabor RF, Grieser F, Dagastine RR, Chan DYC. Measurement and analysis of forces in bubble and droplet systems using AFM. Journal of Colloid and Interface Science. 2012;371(1):1-14.

8. Xie L, Shi C, Cui X, Zeng H. Surface Forces and Interaction Mechanisms of Emulsion Drops and Gas Bubbles in Complex Fluids. Langmuir. 2017;33(16):3911-25.

9. Wang L, Sharp D, Masliyah J, Xu Z. Measurement of Interactions between Solid Particles, Liquid Droplets, and/or Gas Bubbles in a Liquid using an Integrated Thin Film Drainage Apparatus. Langmuir. 2013;29(11):3594-603.

10. Frostad JM, Collins MC, Leal LG. Cantilevered-Capillary Force Apparatus for Measuring Multiphase Fluid Interactions. Langmuir. 2013;29(15):4715-25.

11. Clasohm LY, Connor JN, Vinogradova OI, Horn RG. The "Wimple": Rippled Deformation of a Fluid Drop Caused by Hydrodynamic and Surface Forces during Thin Film Drainage. Langmuir. 2005;21(18):8243-9.

12. Klaseboer E, Chevaillier JP, Gourdon C, Masbernat O. Film Drainage between Colliding Drops at Constant Approach Velocity: Experiments and Modeling. Journal of Colloid and Interface Science. 2000;229(1):274-85.

13. Aveyard R, Binks BP, Cho WG, Fisher LR, Fletcher PDI, Klinkhammer F. Investigation of the Force-Distance Relationship for a Small Liquid Drop Approaching a Liquid-Liquid Interface. Langmuir. 1996;12(26):6561-9.

14. Bremond N, Bibette J. Exploring emulsion science with microfluidics. Soft Matter. 2012;8(41):10549-59.

15. Bremond N, Thiam AR, Bibette J. Decompressing Emulsion Droplets Favors Coalescence. Physical Review Letters. 2008;100(2):024501.

16. Gunes DZ, Clain X, Breton O, Mayor G, Burbidge AS. Avalanches of coalescence events and local extensional flows - Stabilisation or destabilisation due to surfactant. Journal of Colloid and Interface Science.343(1):79-86. 
17. Gunes DZ, Bercy M, Watzke B, Breton O, Burbidge AS. A study of extensional flow induced coalescence in microfluidic geometries with lateral channels. Soft Matter. 2013;9(31):7526-37.

18. Christopher GF, Bergstein J, End NB, Poon M, Nguyen C, Anna SL. Coalescence and splitting of confined droplets at microfluidic junctions. Lab on a Chip. 2009;9(8):1102-9.

19. Borrell $M$, Yoon $Y$, Leal LG. Experimental analysis of the coalescence process via head-on collisions in a time-dependent flow. Physics of Fluids. 2004;16(11):3945-54.

20. Bibette J, Mason TG, Gang H, Weitz DA, Poulin P. Structure of adhesive emulsions. Langmuir. 1993;9(12):3352-6.

21. Bibette J, Calderon FL, Poulin P. Emulsions: basic principles. Reports on Progress in Physics. 1999;62(6):969.

22. Langevin D. Polyelectrolyte and surfactant mixed solutions. Behavior at surfaces and in thin films. Adv Colloid Interface Sci. 2001;89-90(0):467-84.

23. Asnacios A, Langevin D, Argillier J-F. Complexation of Cationic Surfactant and Anionic Polymer at the Air-Water Interface. Macromolecules. 1996;29(23):7412-7.

24. Bergeron V, Langevin D, Asnacios A. Thin-Film Forces in Foam Films Containing Anionic Polyelectrolyte and Charged Surfactants. Langmuir. 1996;12(6):1550-6.

25. Bain CD, Claesson PM, Langevin D, Meszaros R, Nylander T, Stubenrauch C, et al. Complexes of surfactants with oppositely charged polymers at surfaces and in bulk. Advances in Colloid and Interface Science. 2010;155(1):32-49.

26. Nikolov AD, Wasan DT. Ordered micelle structuring in thin films formed from anionic surfactant solutions: I. Experimental. Journal of Colloid and Interface Science. 1989;133(1):1-12.

27. Kumar K, Nikolov AD, Wasan DT. Effect of Film Curvature on Drainage of Thin Liquid Films. Journal of Colloid and Interface Science. 2002;256(1):194-200.

28. Browne C, Tabor RF, Grieser F, Dagastine RR. Direct AFM force measurements between air bubbles in aqueous polydisperse sodium poly(styrene sulfonate) solutions: Effect of collision speed, polyelectrolyte concentration and molar mass. Journal of Colloid and Interface Science. 2015;449:23645.

29. Browne C, Tabor RF, Grieser F, Dagastine RR. Direct AFM force measurements between air bubbles in aqueous monodisperse sodium poly(styrene sulfonate) solutions. Journal of Colloid and Interface Science. 2015;451:69-77.

30. Fewkes CJ, Tabor RF, Dagastine RR. Sphere to rod transitions in self assembled systems probed using direct force measurement. Soft Matter. 2015;11(7):1303-14.

31. Tabor RF, Chan DYC, Grieser F, Dagastine RR. Structural Forces in Soft Matter Systems. J Phys Chem Lett. 2011;2(5):434-7.

32. Tabor RF, Lockie H, Chan DYC, Grieser F, Grillo I, Mutch KJ, et al. Structural forces in soft matter systems: unique flocculation pathways between deformable droplets. Soft Matter. 2011;7(24):1133444.

33. Chiappisi L, Hoffmann I, Gradzielski M. Complexes of oppositely charged polyelectrolytes and surfactants - recent developments in the field of biologically derived polyelectrolytes. Soft Matter. 2013;9(15):3896-909. 
34. Purcell IP, Lu JR, Thomas RK, Howe AM, Penfold J. Adsorption of Sodium Dodecyl Sulfate at the Surface of Aqueous Solutions of Poly(vinylpyrrolidone) Studied by Neutron Reflection. Langmuir. 1998;14(7):1637-45.

35. Prasad M, Palepu R, Moulik SP. Interaction between sodium dodecyl sulfate (SDS) and polyvinylpyrrolidone (PVP) investigated with forward and reverse component addition protocols employing tensiometric, conductometric, microcalorimetric, electrokinetic, and DLS techniques. Colloid and Polymer Science. 2006;284(8):871-8.

36. Chari K, Lenhart WC. Effect of polyvinylpyrrolidone on the self-assembly of model hydrocarbon amphiphiles. Journal of Colloid And Interface Science. 1990;137(1):204-16.

37. Goddard ED. Polymer Surfactant Interaction .1. Uncharged Water-Soluble Polymers and Charged Surfactants. Colloids and Surfaces. 1986;19(2-3):255-300.

38. Goddard ED. Polymer Surfactant Interaction .2. Polymer and Surfactant of Opposite Charge. Colloids and Surfaces. 1986;19(2-3):301-29.

39. Goddard ED. Polymer/surfactant interaction: Interfacial aspects. Journal of Colloid and Interface Science. 2002;256(1):228-35.

40. Clauzel M, Johnson ES, Nylander T, Panandiker RK, Sivik MR, Piculell L. Surface Deposition and Phase Behavior of Oppositely Charged Polyion-Surfactant Ion Complexes. Delivery of Silicone Oil Emulsions to Hydrophobic and Hydrophilic Surfaces. ACS Applied Materials \& Interfaces. 2011;3(7):2451-62.

41. Taylor DJF, Thomas RK, Penfold J. Polymer/surfactant interactions at the air/water interface. Advances in Colloid and Interface Science. 2007;132(2):69-110.

42. Guzmán E, Llamas S, Maestro A, Fernández-Peña L, Akanno A, Miller R, et al. Polymersurfactant systems in bulk and at fluid interfaces. Advances in Colloid and Interface Science. 2016;233(Supplement C):38-64.

43. Nylander T, Samoshina Y, Lindman B. Formation of polyelectrolyte-surfactant complexes on surfaces. Advances in Colloid and Interface Science. 2006;123-126:105-23.

44. Svensson AV, Huang L, Johnson ES, Nylander T, Piculell L. Surface Deposition and Phase Behavior of Oppositely Charged Polyion/Surfactant Ion Complexes. 1. Cationic Guar versus Cationic Hydroxyethylcellulose in Mixtures with Anionic Surfactants. ACS Applied Materials \& Interfaces. 2009;1(11):2431-42.

45. Svensson AV, Johnson ES, Nylander T, Piculell L. Surface Deposition and Phase Behavior of Oppositely Charged Polyion-Surfactant Ion Complexes. 2. A Means to Deliver Silicone Oil to Hydrophilic Surfaces. ACS Applied Materials \& Interfaces. 2010;2(1):143-56.

46. Anthony O, Marques CM, Richetti P. Bulk and Surface Behavior of Cationic Guars in Solutions of Oppositely Charged Surfactants. Langmuir. 1998;14(21):6086-95.

47. Zimin D, Craig VSJ, Kunz W. Adsorption and desorption of polymer/surfactant mixtures at solid-liquid interfaces: Substitution experiments. Langmuir. 2004;20(19):8114-23.

48. Tulpar A, Tilton RD, Walz JY. Synergistic Effects of Polymers and Surfactants on Depletion Forces. Langmuir. 2007;23(8):4351-7.

49. Bremmell KE, Jameson GJ, Biggs S. Forces between surfaces in the presence of a cationic polyelectrolyte and an anionic surfactant. Colloids and Surfaces A: Physicochemical and Engineering Aspects. 1999;155(1):1-10. 
50. Folmer BM, Kronberg B. Effect of Surfactant-Polymer Association on the Stabilities of Foams and Thin Films: Sodium Dodecyl Sulfate and Poly(vinyl pyrrolidone). Langmuir. 2000;16(14):5987-92.

51. Cohen-Addad S, di Meglio J-M. Stabilization of aqueous foam by hydrosoluble polymers. 2 . Role of polymer/surfactant interactions. Langmuir. 1994;10(3):773-8.

52. Gromer A, Penfold R, Gunning AP, Kirby AR, Morris VJ. Molecular basis for the emulsifying properties of sugar beet pectin studied by atomic force microscopy and force spectroscopy. Soft Matter. 2010;6(16):3957-69.

53. Zeng $Y$, von Klitzing R. Structuring of colloidal suspensions confined between a silica microsphere and an air bubble. Soft Matter. 2011;7(11):5329-38.

54. Philip J, Prakash GG, Jaykumar T, Kalyanasundaram P, Mondain-Monval O, Raj B. Interaction between Emulsion Droplets in the Presence of Polymer-Surfactant Complexes. Langmuir. 2002;18(12):4625-31.

55. Fishman M, R. Elrich F. Interactions of aqueous poly(N-vinylpyrrolidone) with sodium dodecyl sulfate. II. Correlation of electric conductance and viscosity measurements with equilibrium dialysis measurements1975. 2740-4 p.

56. Wang G, Olofsson G. Titration Calorimetric Study of the Interaction between Ionic Surfactants and Uncharged Polymers in Aqueous Solution. The Journal of Physical Chemistry B. 1998;102(46):9276-83.

57. Shen $Q$, Wei H, Wang L, Zhou Y, Zhao Y, Zhang Z, et al. Crystallization and Aggregation Behaviors of Calcium Carbonate in the Presence of Poly(vinylpyrrolidone) and Sodium Dodecyl Sulfate. The Journal of Physical Chemistry B. 2005;109(39):18342-7.

58. Wan-Badhi WA, Wan-Yunus WMZ, Bloor DM, Hall DG, Wyn-Jones E. Equilibrium and kinetic studies associated with the interaction between sodium dodecyl sulfate and polyvinylpyrrolidone in aqueous solution. Journal of the Chemical Society, Faraday Transactions. 1993;89(15):2737-42.

59. Zana RL, J.; Lianos, P. Fluorescence probe studies of the aggregation state of sodium dodeculsulfate in aqueous solutions of polioxyethylenegycol and poly- $\mathrm{N}$-vinylpirrolidone. Polym Prepr Am Chem Soc Div Polym Chem. 1982;23(39).

60. Bales BL. A Definition of the Degree of Ionization of a Micelle Based on Its Aggregation Number. The Journal of Physical Chemistry B. 2001;105(29):6798-804.

61. Purcell IP, Thomas RK, Penfold J, Howe AM. Adsorption of SDS and PVP at the air/water interface. Colloids and Surfaces A: Physicochemical and Engineering Aspects. 1995;94(2):125-30.

62. Chari KH, T. Z. Adsorption at the Air/Water Interface from an Aqueous Solution of Poly(vunyl pyrollidone) and Sodium Dodecly Sulfate. J Phys Chem. 1991;95:3302-5.

63. Jones $M N$. The interaction of sodium dodecyl sulfate with polyethylene oxide. Journal of Colloid and Interface Science. 1967;23(1):36-42.

64. Lange H. Wechselwirkung zwischen Natriumalkylsulfaten und Polyvinylpyrrolidon in wäßrigen Lösungen. Kolloid-Zeitschrift und Zeitschrift für Polymere. 1971;243(2):101-9.

65. Fadnavis N, Engberts JBFN. Surfactant-polymer interactions and their effects on the micellar inhibition of the neutral hydrolysis of 1-benzoyl-1,2,4-triazole. Journal of the American Chemical Society. 1984;106(9):2636-40. 
66. Öztekin N, Erim FB. Determination of Critical Aggregation Concentration in the Poly(vinylpyrrolidone)-Sodium Dodecyl Sulfate System by Capillary Electrophoresis. Journal of Surfactants and Detergents. 2013;16(3):363-7.

67. Chen J, Gong X, Yang H, Yao Y, Xu M, Chen Q, et al. NMR Study on the Effects of Sodium nDodecyl Sulfate on the Coil-to-Globule Transition of Poly( $\mathrm{N}$-isopropylacrylamide) in Aqueous Solutions. Macromolecules. 2011;44(15):6227-31.

68. Francois J, Dayantis J, Sabbadin J. Hydrodynamical behaviour of the poly(ethylene oxide)sodium dodecylsulphate complex. European Polymer Journal. 1985;21(2):165-74.

69. Xia J, Dubin PL, Kim Y. Complex formation between poly(oxyethylene) and sodium dodecyl sulfate micelles: light scattering, electrophoresis, and dialysis equilibrium studies. The Journal of Physical Chemistry. 1992;96(16):6805-11.

70. Jiang WH, Han SJ. Viscosity of Nonionic Polymer/Anionic Surfactant Complexes in Water. Journal of Colloid and Interface Science. 2000;229(1):1-5.

71. Sorci GA, Reed WF. Electrostatic and Association Phenomena in Aggregates of Polymers and Micelles. Langmuir. 2002;18(2):353-64.

72. Minatti E, Norwood DP, Reed WF. Surfactant/Polymer Assemblies. 2. Polyelectrolyte Properties. Macromolecules. 1998;31(9):2966-71.

73. Wu Y, Chen J, Fang Y, Zhu M. Polyvinylpyrrolidone-sodium dodecylsulfate complex is a family of pseudo-polyanions with different charge densities: Evidence from capillary electrophoresis, capillary viscosimetry and conductometry. Journal of Colloid and Interface Science. 2016;479:34-42.

74. Zhang X, Manica R, Tchoukov P, Liu Q, Xu Z. Effect of Approach Velocity on Thin Liquid Film Drainage between an Air Bubble and a Flat Solid Surface. The Journal of Physical Chemistry C. 2017;121(10):5573-84.

75. Frostad JM, Walter J, Leal LG. A scaling relation for the capillary-pressure driven drainage of thin films. Physics of Fluids. 2013;25(5):052108.

76. Shen B, Ricouvier J, Malloggi F, Tabeling P. Designing Colloidal Molecules with Microfluidics. Advanced Science. 2016;3(6):1600012-n/a.

77. Berry JD, Neeson MJ, Dagastine RR, Chan DYC, Tabor RF. Measurement of surface and interfacial tension using pendant drop tensiometry. Journal of Colloid and Interface Science. 2015;454:226-37.

78. Hutter JL, Bechhoefer J. Calibration of Atomic-Force Microscope Tips. Rev Sci Instrum. 1993;64(7):1868-73.

79. Manor O, Vakarelski IU, Tang X, O'Shea SJ, Stevens GW, Grieser F, et al. Hydrodynamic Boundary Conditions and Dynamic Forces between Bubbles and Surfaces. Phys Rev Lett. 2008;101(2):024501.

80. Dagastine RR, Prieve DC, White LR. Forces between a rigid probe particle and a liquid interface III. Extraction of the planar half-space interaction energy $E(D)$. Journal of Colloid and Interface Science. 2004;269(1):84-96.

81. Israelachvili JN. Intermolecular and Surface Forces San Diego: Academic Press; 1991.

82. Asakura S, Oosawa F. On Interaction between Two Bodies Immersed in a Solution of Macromolecules. J Chem Phys. 1954;22(7):1255-6. 
83. Chan DYC, Dagastine RR, White LR. Forces between a Rigid Probe Particle and a Liquid Interface. I. The Repulsive Case. Journal of Colloid and Interface Science. 2001;236(1):141-54.

84. Dagastine RR, White LR. Forces between a Rigid Probe Particle and a Liquid Interface. II. The General Case. Journal of Colloid and Interface Science. 2002;247(2):310-20.

85. Chan DYC, Klaseboer E, Manica R. Theory of non-equilibrium force measurements involving deformable drops and bubbles. Advances in Colloid and Interface Science. 2011;165(2):70-90.

86. Chan DYC, Klaseboer E, Manicac R. Dynamic deformations and forces in soft matter. Soft Matter. 2009;5:2858-61.

87. Bhatt D, Newman J, Radke CJ. Equilibrium Force Isotherms of a Deformable Bubble/Drop Interacting with a Solid Particle across a Thin Liquid Film. Langmuir. 2001;17(1):116-30.

88. Carnie SL, Chan DYC, Lewis C, Manica R, Dagastine RR. Measurement of Dynamical Forces between Deformable Drops Using the Atomic Force Microscope. I. Theory. Langmuir. 2005;21(7):2912-22.

89. Tabor RF, Lockie H, Mair D, Manica R, Chan DYC, Grieser F, et al. Combined AFM-Confocal Microscopy of Oil Droplets: Absolute Separations and Forces in Nanofilms. The Journal of Physical Chemistry Letters. 2011;2:961-5.

90. van Steijn V, Korczyk PM, Derzsi L, Abate AR, Weitz DA, Garstecki P. Block-and-break generation of microdroplets with fixed volume. Biomicrofluidics. 2013;7(2):024108.

91. Mészáros R, Varga I, Gilányi T. Effect of Polymer Molecular Weight on the Polymer/Surfactant Interaction. The Journal of Physical Chemistry B. 2005;109(28):13538-44.

92. Milling AJ. Depletion and Structuring of Sodium Poly(styrenesulfonate) at the Silica-Water Interface. J Phys Chem. 1996;100(21):8986-93.

93. Milling AJ, Kendall K. Depletion, Adsorption, and Structuring of Sodium Poly(acrylate) at the Water-Silica Interface. 1. An Atomic Force Microscopy Force Study. Langmuir. 2000;16(11):5106-15.

94. Biggs S. Direct measurement of the depletion interaction in binary solutions of polyelectrolytes. Physical Chemistry Chemical Physics. 2010;12(16):4172-7.

95. Biggs S, Burns JL, Yan Yd, Jameson GJ, Jenkins P. Molecular Weight Dependence of the Depletion Interaction between Silica Surfaces in Solutions of Sodium Poly(styrene sulfonate). Langmuir. 2000;16(24):9242-8.

96. Biggs S, Dagastine RR, Prieve DC. Oscillatory Packing and Depletion of Polyelectrolyte Molecules at an Oxide-Water Interface. Journal of Physical Chemistry B. 2002;106(44):11557-64.

97. Dobrynin AV, Colby RH, Rubinstein M. Scaling Theory of Polyelectrolyte Solutions. Macromolecules. 1995;28(6):1859-71.

98. Nespolo SA, Bevan MA, Chan DYC, Grieser F, Stevens GW. Hydrodynamic and Electrokinetic Properties of Decane Droplets in Aqueous Sodium Dodecyl Sulfate Solutions. Langmuir. 2001;17(23):7210-8.

99. Dobrynin AV, Rubinstein M. Theory of polyelectrolytes in solutions and at surfaces. Progress in Polymer Science. 2005;30(11):1049-118.

100. Edwards TD, Bevan MA. Depletion-Mediated Potentials and Phase Behavior for Micelles, Macromolecules, Nanoparticles, and Hydrogel Particles. Langmuir. 2012;28(39):13816-23. 
101. Braem AD, Prieve DC, Tilton RD. Electrostatically Tunable Coadsorption of Sodium Dodecyl Sulfate and Poly(ethylene oxide)-b-poly(propylene oxide)-b-poly(ethylene oxide) Triblock Copolymer to Silica. Langmuir. 2001;17(3):883-90.

102. Cattoz B, Cosgrove T, Crossman M, Prescott SW. Surfactant-Mediated Desorption of Polymer from the Nanoparticle Interface. Langmuir. 2012;28(5):2485-92.

103. Manor O, Chau TT, Stevens GW, Chan DYC, Grieser F, Dagastine RR. Polymeric Stabilized Emulsions: Steric Effects and Deformation in Soft Systems. Langmuir. 2012;28(10):4599-604.

104. Alvarez NJ, Walker LM, Anna SL. Diffusion-limited adsorption to a spherical geometry: The impact of curvature and competitive time scales. Physical Review E. 2010;82(1).

105. Ma S, Sherwood JM, Huck WTS, Balabani S. On the flow topology inside droplets moving in rectangular microchannels. Lab on a Chip. 2014;14(18):3611-20. 
Supporting information for "Forces between oil drops in polymer-surfactant systems: linking direct force measurements to microfluidic observations"

1 Microfluidic Design Schematics and Dimensions
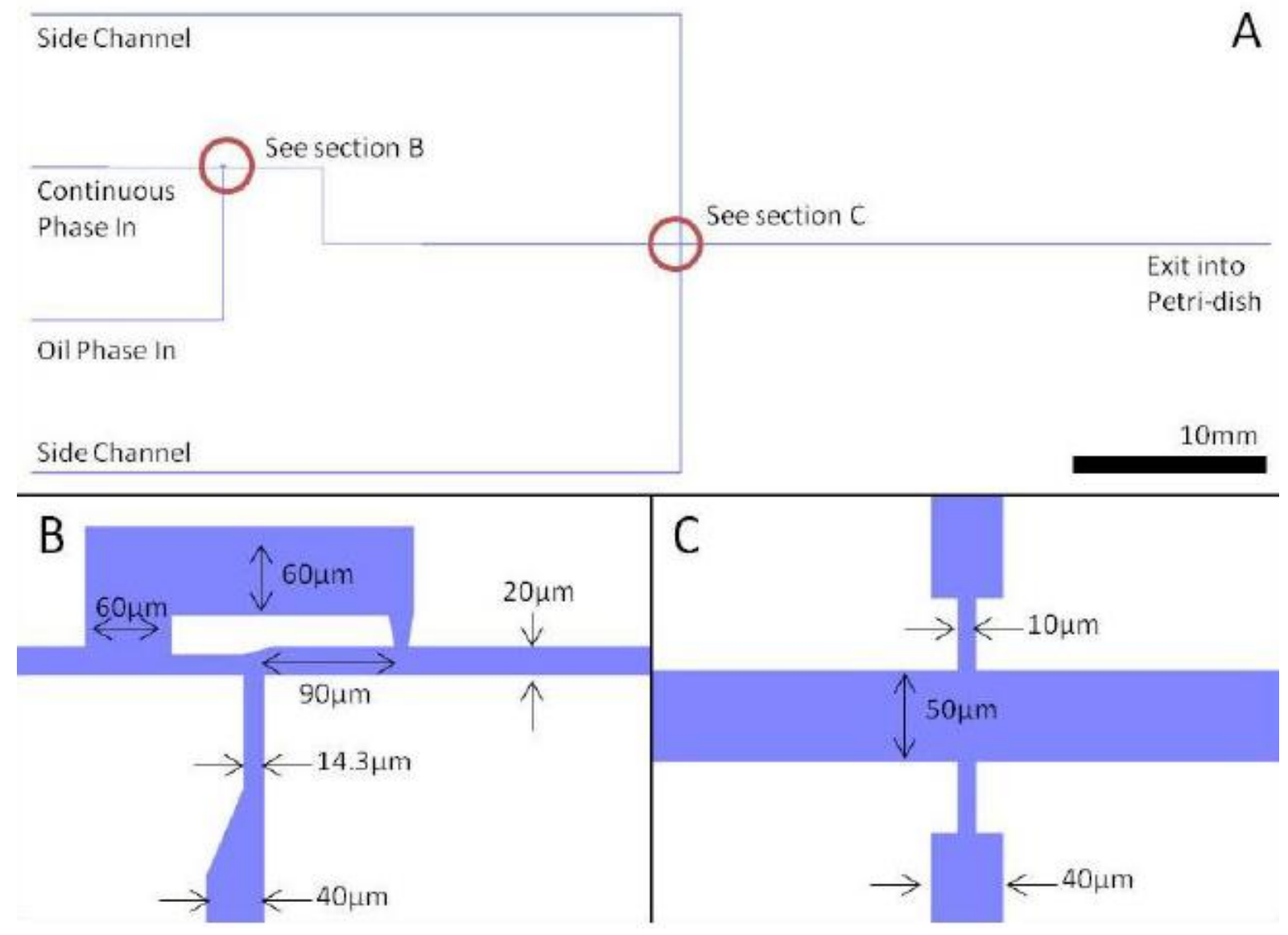

Figure S1 Schematic diagram of the microfluidic design used for drop chaining experiments showing (A) an overview of the device from entry to exit, (B) the dimensions and geometry of the block and break design, and $(\mathbf{C})$ the dimensions and geometry of the exit channels for removal of excess aqueous solution. 


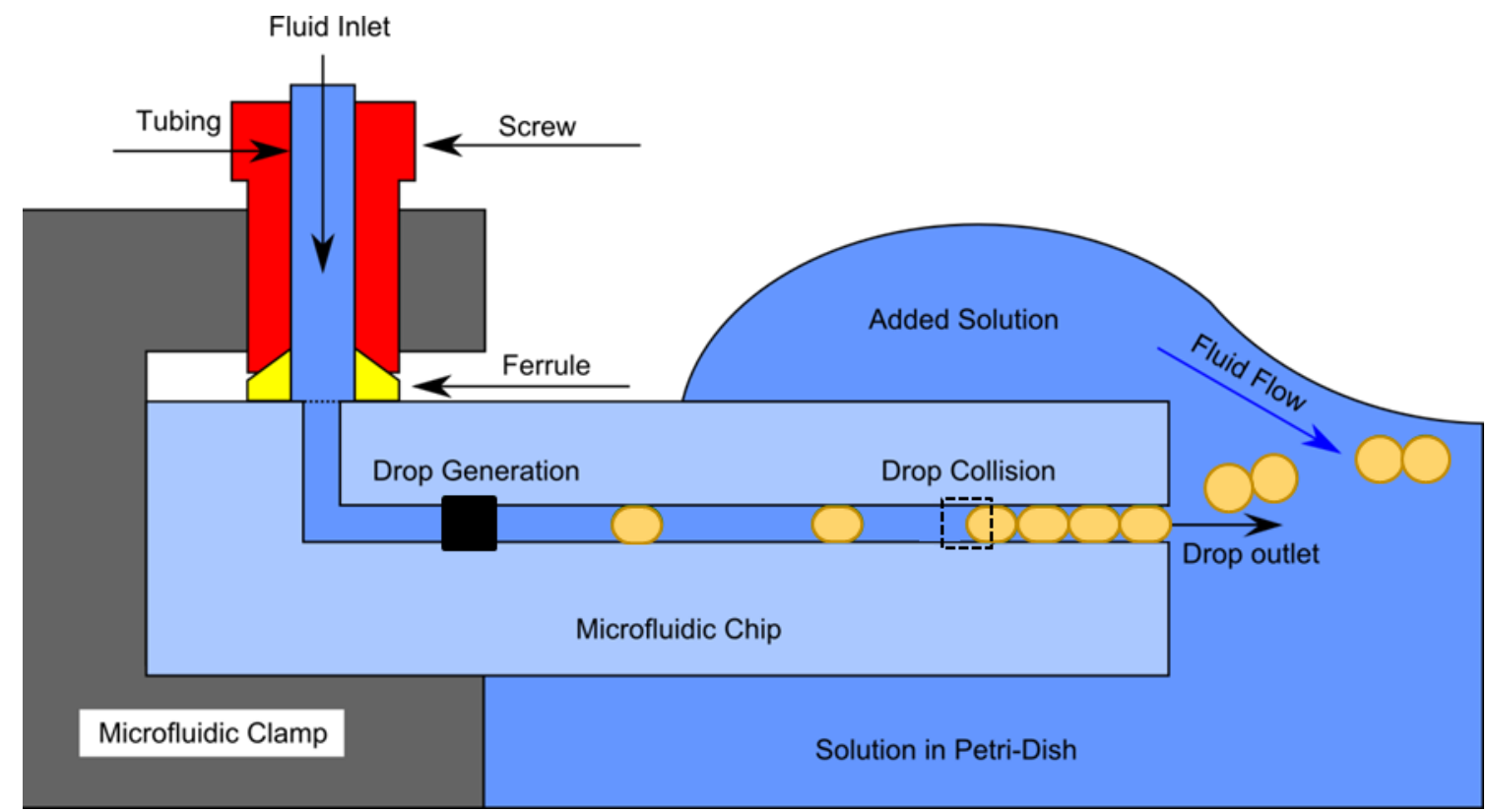

Figure S2 : Side on schematic diagram of the microfluidic apparatus and surrounding fluid designed to generate drop chains. After the drops are generated and collided within the microfluidic device the droplet chains flow into solution. Additional solution is added on the top of the microfluidic device and flows over the exit port, mitigating against drop aggregation at the exit of the microfluidic device. 


\section{Stokes-Reynolds-Young-Laplace (SRYL) model}

The SRYL-model is a well described method that matches an inner numerical solution, which encompasses the drop or bubble interaction zone, to an outer analytical solution for the drop or bubble profile. The governing equation are solved numerically using four boundary conditions and one initial condition to find the shape of the film, total pressure and force over the entire droop-drop interaction. Two of the four boundary conditions occur in the inner region at $r=0$, where it is assumed that the total pressure $\mathrm{P}$, and gradient of the film is zero throughout the interaction, giving the boundary conditions:

$$
\begin{aligned}
& \left.\frac{\partial P(r, t)}{\partial r}\right|_{r=0, t}=0 \\
& \left.\frac{\partial h(r, t)}{\partial r}\right|_{r=0, t}=0
\end{aligned}
$$

Outside the interaction zone it is expected that the drops or bubbles have similar behaviour to rigid particles. Therefore, the pressure is inversely proportional to $r^{-4}$ as $r \rightarrow \infty$ such that the third boundary condition becomes:

$$
\left.\frac{\partial P(r, t)}{\partial r}\right|_{r=r_{\max }, t}=-\frac{4 P(r, t)}{r^{4}}
$$

Where the inner and outer regions meet $\left(r=r_{\max }\right)$ it is assumed that the analytical approximation holds true, resulting in the final condition:

$$
\begin{aligned}
\left.\frac{\partial h(r, t)}{\partial t}\right|_{r=r_{\text {max }}, t}= & -\frac{d \Delta X(t)}{d t}+\frac{1}{K} \frac{d F(t)}{d t}-\frac{1}{2 \pi \gamma} \\
& \times \frac{d F(t)}{d t}\left(\log \left(\frac{\mathrm{R}_{2}}{R_{1}}\right)+B\left(\theta_{1}\right)-B\left(\theta_{2}\right)\right)
\end{aligned}
$$

Here $\Delta X$ is the displacement of the cantilever piezo head, $\mathrm{K}$ is the cantilever spring constant, $\theta_{1}$ and $\theta_{2}$ are the contact angles of drops on the cantilever and substrate respectively, and

$$
\mathrm{B}(\theta)=1+\frac{1}{2} \log \left(\frac{1+\cos \theta}{1-\cos \theta}\right)
$$

For a pinned contact line or,

$$
\mathrm{B}(\theta)=1+\frac{1}{2} \log \left(\frac{1+\cos \theta}{1-\cos \theta}\right)-\frac{1}{2+\cos \theta}
$$

For a constant contact angle. To complete the model, it is predicted that the drops are far enough apart in their initial separation that neither body is deformed, giving the films a parabolic shape and yielding the initial condition:

$$
h(r, \mathrm{t}=0)=h_{0}+\frac{r^{2}}{\mathrm{R}}
$$

Where $h_{0}$ is the initial separation between the two drops at $r=0$. 


\section{AFM force curves between two hydrophilic silica surfaces in solutions of PVP and SDS}

The force between silica surfaces has been well studied in the literature as a model hydrophilic system, which is predominant in many colloidal probe measurements. Figure S2A shows direct force measurements between a $2.5 \mu \mathrm{m}$ radii silica particle and glass slide in solutions of $0.5 \%$ PVP and $2 \mathrm{mM}$, $10 \mathrm{mM}$ and $30 \mathrm{mM}$ SDS. These curves show behaviours consistent with the presence of a steric brush, with increasing repulsion at close separation. Despite the potential for polymer bridging, it is important to note that there is no evidence of an adhesive force or hysteresis on the retract curve that would indicate bridging over the three concentrations. With increasing concentration, the slope of the curve increases, signifying an increase in brush length.

PVP and SDS have been shown in the literature to absorb at the silica-water interface as a complex brush $(45,46)$. Prescott et al. provided evidence of a three-stage binding process with increasing surfactant concentration, essentially comprising of initial surfactant binding, chain elongation and finally desorption of the adsorbed complex. From SANS and NMR experiments they demonstrate that around the CAC ( $\sim 2.5 \mathrm{mM})$ surfactants begin to aggregate to the adsorbed polymer, and at SDS concentrations above the $\mathrm{CMC}$ there is a barely discernible presence of a layer, concluding that desorption of the complex had occurred.

The steric forces were modelled using the potential energy between parallel plates of separation $h$ with adsorbed polymer brushes of size $L_{o}$ according to the de Gennes model $(1,2)$ :

$$
E_{\text {Steric }}=\left\{\begin{array}{l}
\frac{8 k_{b} T L_{o}}{35 s^{3}}\left[7\left(\frac{2 L_{o}}{h}\right)^{\frac{5}{4}}-5\left(\frac{h}{2 L_{o}}\right)^{\frac{7}{4}}-12\right] \\
0 \quad \text { for } h<2 L_{o} \\
\text { for } h 2 L_{o}
\end{array}\right.
$$

Using the Derjaguin approximation for a sphere and infinite surface, a model was fitted to the experimental data to extract the polymer brush length at each SDS concentration. Figure S2B shows the calculated polymer brush lengths at each SDS concentration. These results are in strong agreement with those obtained by Prescott et al. (3).

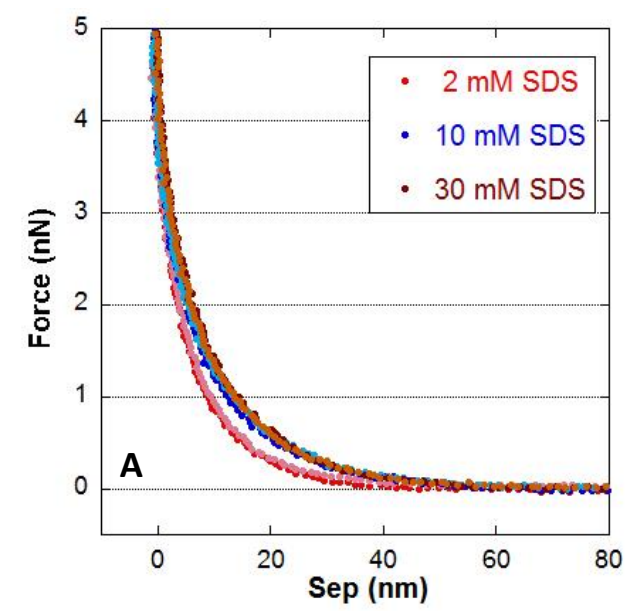

B
\begin{tabular}{|l|l|}
\hline SDS Concentration (mM) & Brush Length $(\mathbf{n m})$ \\
\hline 2 & $22.6 \pm 0.3$ \\
\hline 5 & $24.6 \pm 0.6$ \\
\hline 10 & $29.1 \pm 0.3$ \\
\hline 15 & $29.5 \pm 0.2$ \\
\hline 20 & $30.2 \pm 0.4$ \\
\hline 30 & $28.84 \pm 0.6$ \\
\hline
\end{tabular}

Figure S3 (A) The approach and retract AFM force curves between a silica particle and glass slide in an aqueous solutions of $0.5 \%$ PVP with $2 \mathrm{mM}$ SDS (red), $10 \mathrm{mM}$ SDS (blue) and $30 \mathrm{mM}$ SDS (brown) (B) Summary of the complex brush length determined by fitting a de Gennes model to the AFM curve data. 


\section{Surfactant molecule mass balance - determining solution ionic strength}

Zana et al. showed through florescence studies of a SDS and PVP system that polymer bound micelles increase in aggregation number from $18-50$ as the SDS concentration increases from 10 to $30 \mathrm{mM}$ (56). Surfactants in micelles have an 'effective degree of counterion disassociation' ( $\alpha$ ), with Bales et al. reporting a value of 0.272 for SDS over a range of salt concentrations (57). However, the degree of disassociation is also dependent on the aggregation number of the micelle. Therefore, Wan-Badhi et al. used electrochemical measurements and gel filtration to provide the counterion disassociation as a function of surfactant concentration for a PVP-SDS system, recording a decrease from 0.45 to 0.27 with increasing SDS concentration from 10 to $30 \mathrm{mM}$. They also predict that one polymer molecule is in contact with approximately 40 micelles at saturation of a $700000 \mathrm{MW}$ PVP polymer, giving approximately 158 PVP monomers per micelle (55).

Based on the above studies, setting the salt concentration of the solution is equal to the summation of the contribution of disassociated ions from polymer bound SDS micelles (subscript p), free SDS molecules in solution (subscript s) and free SDS micelles (subscript $\mathrm{m}$ ), which can be represented by the following equation:

$$
[\text { Salt }]=\alpha_{p}\left[S D S_{p}\right]+\left[S D S_{S}\right]+\alpha_{m}\left[S D S_{m}\right]
$$

The number of PVP monomers per SDS molecule can be calculated from the number of SDS molecules per micelle (obtained from Zana et al.) and the number of PVP monomers per micelle (obtained from Wan-Badhi et al.), assuming that the number of bound micelles remains constant and the aggregation number alone increases with increasing SDS concentration. Using the PVP monomer amount of 4.5 mmol for a $0.5 \mathrm{wt} \%$ PVP solution, it is then possible to calculate the amount of SDS bound to the PVP as summarised in Table S1.

Table S1 Summary of variables for the calculation of polymer bound SDS molecules in different solutions of SDS and PVP

\begin{tabular}{|l|l|l|l|l|}
\hline SDS concentration $(\mathrm{mM})$ & 10 & 15 & 20 & 30 \\
\hline SDS amount $(\mathrm{mmol})$ & 1 & 1.5 & 2 & 3 \\
\hline PVP monomer/ micelle & 158 & 158 & 158 & 158 \\
\hline SDS molecule/ micelle & 18 & 30 & 38 & 50 \\
\hline PVP monomer/ SDS molecule & 8.76 & 5.26 & 4.15 & 3.15 \\
\hline SDS on PVP $(\mathrm{mmol})$ & 0.51 & 0.86 & 1.09 & 1.43 \\
\hline SDS on PVP $(\mathrm{mM})\left[\boldsymbol{S D} \boldsymbol{S}_{\boldsymbol{p}}\right]$ & 5.14 & 8.57 & 10.9 & 14.3 \\
\hline
\end{tabular}

Deducting the concentration of polymer bound SDS from the total amount of SDS added to solution gives the total amount of SDS free in solution. As the CMC of SDS is $\approx 8 \mathrm{mM}$, free surfactant concentrations above this value are considered to be in the form of micelles, thus contributing to the free micelle concentration. Table S2 details these values, the disassociation factors $(\alpha)$ and final salt concentrations for each of the solutions. 
Table S2 Summary of variables for the calculation of total electrolyte concentration in different solutions of SDS and PVP

\begin{tabular}{|l|l|l|l|l|}
\hline SDS concentration $(\mathrm{mM})$ & 10 & 15 & 20 & 30 \\
\hline SDS free in solution $(\mathrm{mM})$ & 4.86 & 6.43 & 9.14 & 15.7 \\
\hline Free SDS molecules $(\mathrm{mM})\left[\boldsymbol{S D} \boldsymbol{S}_{\boldsymbol{s}}\right]$ & 4.86 & 6.43 & 8 & 8 \\
\hline Free SDS micelles $(\mathrm{mM})\left[\boldsymbol{S D} \boldsymbol{S}_{\boldsymbol{m}}\right]$ & - & - & 1.14 & 7.71 \\
\hline $\begin{array}{l}\text { Polymer bound micelle } \\
\text { disassociation factor }\left[\boldsymbol{\alpha}_{\boldsymbol{p}}\right]\end{array}$ & 0.45 & 0.27 & 0.27 & 0.27 \\
\hline $\begin{array}{l}\text { Free SDS micelle disassociation } \\
\text { factor }\left[\boldsymbol{\alpha}_{\boldsymbol{p}}\right]\end{array}$ & 0.27 & 0.27 & 0.27 & 0.27 \\
\hline Salt $(\mathrm{mM})$ & 7.17 & 8.74 & 11.24 & 13.94 \\
\hline $\begin{array}{l}\text { Concentration of additional } \\
\text { Electrolyte }\end{array}$ & 4.86 & 6.43 & 8.31 & 10.1 \\
\hline
\end{tabular}

To apply scaling theory analysis the concentration of additional electrolyte is also required. This is simply the sum of the ions contributed by the free SDS molecules and free SDS micelles, as shown in Table S2.

\section{Fitting parameters for deformable force measurements}

Table S3 Summary of fitting variables used in and obtained from fitting equilibrium force curves between two tetradecane drops while in the presence of $0.5 \mathrm{wt} \%$ PVP and varying SDS concentrations

\begin{tabular}{|l|l|l|l|l|}
\hline SDS concentration $(\mathrm{mM})$ & 10 & 15 & 20 & 30 \\
\hline Interfacial Tension $(\mathrm{mN} / \mathrm{m})$ & $16.4 \pm 3$ & $13.4 \pm 3$ & $11.1 \pm 3$ & $8.5 \pm 3$ \\
\hline Drop-Substrate Radii $(\mu \mathrm{m})$ & $28.5 \pm 2$ & $28 \pm 2$ & $28 \pm 2$ & $27.2 \pm 2$ \\
\hline Drop-Cantilever Radii $(\mu \mathrm{m})$ & $37.2 \pm 2$ & $33.5 \pm 2$ & $33.5 \pm 2$ & $23.2 \pm 2$ \\
\hline Drop-Substrate Contact Angle $(\theta)$ & $124.3 \pm 5$ & $130.8 \pm 5$ & $130.8 \pm 5$ & $130.9 \pm 5$ \\
\hline Drop-Cantilever Contact Angle $(\theta)$ & $137.5 \pm 5$ & $131.7 \pm 5$ & $131.7 \pm 5$ & $139.7 \pm 5$ \\
\hline Spring Constant $(\mathrm{N} / \mathrm{m})$ & 0.132 & 0.132 & 0.132 & 0.282 \\
\hline Ionic Strength $(\mathrm{mM})$ & $7.2 \pm 0.3$ & $9.1 \pm 0.7$ & $11.7 \pm 0.5$ & 13.94 \\
\hline Calculated Debye Length $(\mathrm{nm})$ & 3.6 & 3.2 & 2.8 & 2.6 \\
\hline Rigid Fit Surface Potential $(\mathrm{mV})$ & $15 \pm 2$ & $15 \pm 2$ & $15 \pm 2$ & $16 \pm 2$ \\
\hline Rigid Fit Depletion Length $(\mathrm{nm})$ & $12.8 \pm 0.9$ & $11.5 \pm 0.8$ & $11.4 \pm 0.6$ & $9.7 \pm 1.0$ \\
\hline Rigid Fit Osmotic Pressure $(\mathrm{Pa})$ & $667 \pm 65$ & $572 \pm 51$ & $738 \pm 63$ & $1117 \pm 109$ \\
\hline Fitted Surface Potential $(\mathrm{mV})$ & $26 \pm 2$ & $14 \pm 2$ & $23 \pm 2$ & $14 \pm 2$ \\
\hline
\end{tabular}


6 AFM forces curve of 0.5 wt\% PVP and no added SDS

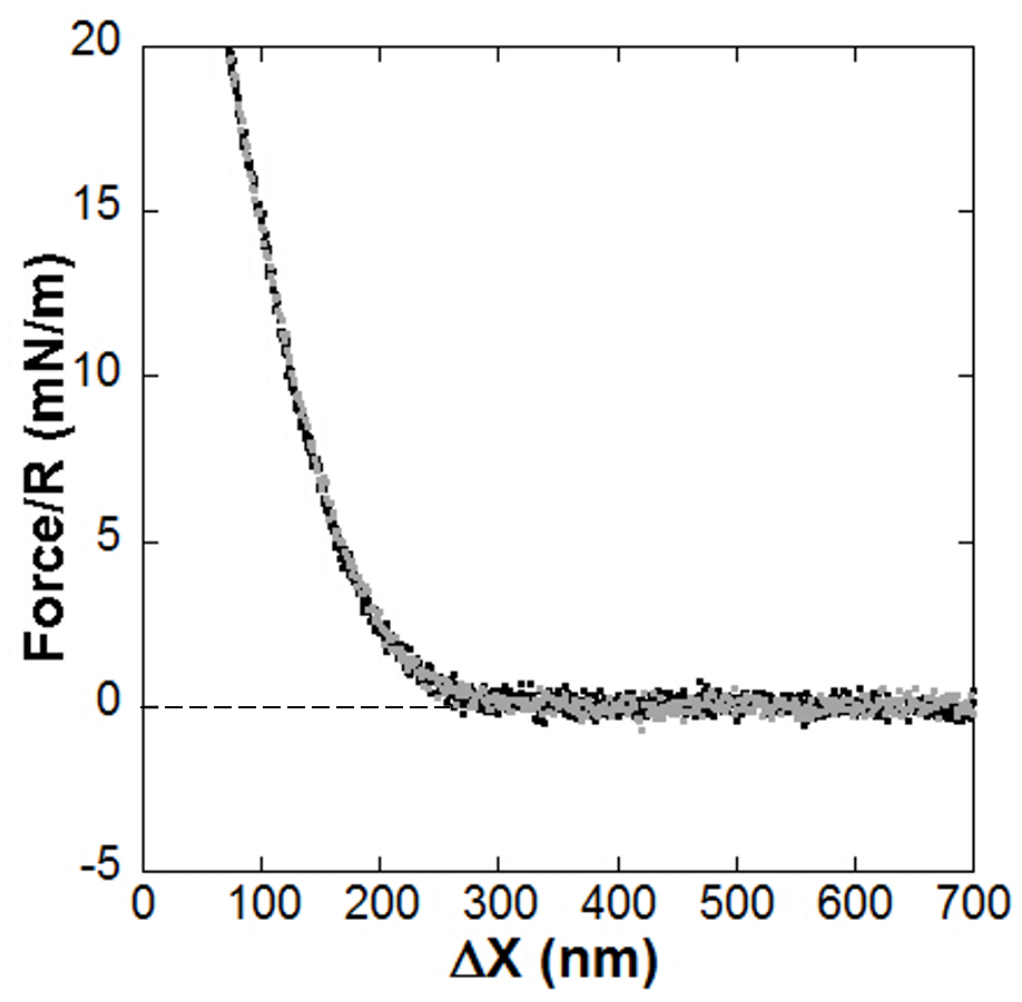

Figure S4 Force versus separation for the interaction between an $82 \mu \mathrm{m}$ and $75 \mu \mathrm{m}$ diameter TD drop in 0.5 wt\% PVP solution, showing the approach (grey) and retract (black) from an AFM measurement.

\section{References}

1. de Gennes PG. Polymers at an interface; a simplified view. Adv Colloid Interface Sci. 1987;27(34):189-209.

2. Alexander S. Adsorption of chain molecules with a polar head a scaling description. J Phys (Paris) FIELD Full Journal Title:Journal de Physique (Paris). 1977;38(8):983-7.

3. Cattoz B, Cosgrove T, Crossman M, Prescott SW. Surfactant-Mediated Desorption of Polymer from the Nanoparticle Interface. Langmuir. 2012;28(5):2485-92. 


\section{University Library}

\section{- M M I N E R VA A gateway to Melbourne's research publications}

Minerva Access is the Institutional Repository of The University of Melbourne

\section{Author/s:}

Jamieson, EJ;Fewkes, CJ;Berry, JD;Dagastine, RR

Title:

Forces between oil drops in polymer-surfactant systems: Linking direct force measurements to microfluidic observations

\section{Date:}

2019-05-15

\section{Citation:}

Jamieson, E. J., Fewkes, C. J., Berry, J. D. \& Dagastine, R. R. (2019). Forces between oil drops in polymer-surfactant systems: Linking direct force measurements to microfluidic observations. Journal of Colloid and Interface Science, 544, pp.130-143. https:// doi.org/10.1016/j.jcis.2019.02.051.

Persistent Link:

http://hdl.handle.net/11343/243039 